\title{
Regularization Methods Applied to Noisy Response from Beams under Static Loading
}

\author{
Miguel Casero ${ }^{1}$, Enrique Covián ${ }^{2}$, and Arturo González ${ }^{3}$
}

\author{
${ }^{1}$ School of Civil Engineering, University College Dublin, Newstead, Belfield, Dublin 4, Ireland, email \\ (corresponding author): miguel.caseroflorez@ucd.ie \\ ${ }^{2}$ Mieres Polytechnic School, University of Oviedo, C/ Gonzalo Gutiérrez Quirós s/n 33600, Mieres, Spain, \\ email: covianenrique@uniovi.es \\ ${ }^{3}$ School of Civil Engineering, University College Dublin, Newstead, Belfield, Dublin 4, Ireland, email: \\ arturo.gonzalez@ucd.ie
}

\section{Abstract}

The estimation of flexural stiffness from static loading test data is the basis of many methods assessing the condition of structural elements. These methods are usually developed under the assumption of having sufficiently accurate data available. Hence, their performance deteriorates as the differences between the measured and true values of the response, often denoted as noise, increase. The proposed methodology is specifically designed to mitigate errors derived from noisy static data when estimating flexural stiffness. It relies on the linearization of the equations relating displacements to stiffness through the unit-force theorem, combined with regularization tools such as L-curve and generalized cross-validation. The methodology is tested using theoretical simulations of the static response of a simply supported beam subjected to a 4-point flexural test for several levels of noise, two types of responses (deflections and rotations) and different levels of discretization. Recommendations for selecting the optimal regularization tool and parameter are provided. The use of rotations as inputs for predicting stiffness is shown to outperform deflections. Finally, the methodology is extended to a statically indeterminate beam.

Keywords: regularization methods; noisy measures; static test; L-curve; generalized crossvalidation $(G C V)$ 


\section{Introduction}

While dynamic measurements are typically preferred for structural health monitoring purposes, static measurements have been more common in diagnostic and proof loading tests. On the one hand, dynamic measurements are able to capture features of the structure (i.e., frequencies, mode shapes or damping) without the need to exactly know the load imposed on the structure. Therefore, a significant change in these dynamic features can be a sign of deterioration, or of a variation in environmental and operational loads. On the other hand, static tests usually employ a load of known magnitude and position on the structure to accurately estimate structural stiffness from the measures or to ensure that the maximum response is below a specified threshold. A number of methods are proposed in the literature to assess the condition of a structure from static test data. They typically involve estimating the stiffness of the structure from simulated or measured displacement inputs through static equilibrium, i.e., the vector of the displacement response is related to the vector of applied forces at the Degrees of Freedom of a Finite Element (FE) model via the stiffness matrix. In simple cases, the equation for the deflection of a beam is used instead of an FE approach. Sanayei and Scampoli (1991), Bakhtiari-Nejad et al (2005) and Abdo (2012) have demonstrated how changes in displacement curvature, changes in the force vector from damaged and undamaged scenarios, and changes in the stiffness matrix respectively can be used for detecting structural damage.

Overall, methods such as Hjelmstad and Shin (1997), Terlaje and Truman (2007), or Caddemi and Morassi (2007) fit measures or simulated displacements to the response provided by numerical or FE equations. The latter develop closed formulas for the detection of cracks in beams through direct analysis of the variation of deflection due to damage. The same authors later extend their work to the detection of multiple cracks in the same beam (Caddemi and Morassi 2011). Yang and Sun (2010) solve the location and quantification of damage separately, achieving the first goal through flexibility disassembly theory. Other authors 
estimate the distribution of stiffness throughout the structure using observability techniques or optimization algorithms to the available static measures. The equations of static equilibrium derived from the FE method lead to a non-linear system where stiffness are unknowns. Tomas et al (2018) apply observability to this system for establishing the subset of structural parameters that can be identified with a given subset of measures. The problem of finding the stiffness for each element is addressed via an optimization cross-entropy algorithm in Walsh and González (2009) and González et al (2013) for a beam and in Walsh et al (2010) for a plate model. This algorithm involves an iterative approach that minimizes an objective function based on the difference between the simulated/measured displacements and those calculated from a discretized FE model of the structure.

A problem identified in most model-based methods is the overfitting of the objective function to the input data. These are affected by noise and, therefore, the best-fit of the calculated and experimental displacements leads to stiffness profiles that are not feasible in practice (i.e., in some cases, they could even lead to negative values of stiffness). For instance, as the crossentropy algorithm by Walsh and González (2009) progresses while seeking to minimize the objective function, the optimal shape can only be approximated through high and unrealistic variations of stiffness between close discretized elements, even for relatively high signal to noise ratios, with errors near the supports being particularly significant (Walsh and González 2009, González et al 2013). However, the stiffness of a true beam will typically vary smoothly except in particular instances such as sudden changes in geometry. In a real scenario of a concrete beam, stiffness loss will be maximum at the location of a crack/damage and then, it will decrease gradually until becoming zero at about one and a half times the beam depth to each side of the crack (Sinha et al 2002).

Noise is a broad term that encompasses inaccuracies in measures (i.e., lack of resolution), environmental and electromagnetic noise, among others. Noise is often considered to be 
particularly relevant in dynamic tests, i.e., Hester and González (2015) measure relative errors of $2.5 \%$ for a QA700 accelerometer purely at rest. Although noise will typically be of smaller magnitude in static measures, its impact still needs to be taken into account (Dewangan and Barai 2012, Ma and Solís 2017, Ok et al 2018). In the case of civil infrastructure, differences in deflections between healthy and damaged stages may be at the millimetric or sub-millimetric level when aiming to capture damage at an early stage. Precisions of 1 to $4 \mathrm{~mm}$ (Pera and Ferrando 2017), $0.2 \mathrm{~mm}$ (Sztubecki et al 2016), $0.001 \mathrm{~mm}$ (González-Jorge et al 2012, Mosalam et al 2014), and 0.01 to $0.04 \mathrm{~mm}$ (Khuc and Catbas 2017) have been reported for deflections measured with laser scanners, total stations, displacement transducers and digital cameras respectively. For a $1 \mathrm{~mm}$ deflection, the resolution will cause between $0.1 \%$ and $4 \%$ noise for displacement transducers and digital cameras. It must be noted that the impact of noise on static measures will not be uniform throughout a structure, and that noise will have a more severe effect the smaller the measured response. In a simply supported beam, the relative error in deflection measures due to noise will be higher near the supports than at midspan. As a result, the accurate estimation of the distribution of stiffness becomes a challenging problem that is highly influenced by the location and number of available measures, noise and the level of discretisation of the model. If rotations were employed instead of deflections, resolutions of 0.0014 rad have been obtained via direct measurement with inclinometers (Ha et al 2013). In practice, the exact relative noise will depend on the sensor specification, the distance from the measuring sensor to the measurement point, and the exact amount of measured deflection or rotation - which will vary with the location of the measurement point, the position and magnitude of the load and the boundary conditions and stiffness distribution of the structure, etc.

Previous methods have proven to be successful when sufficiently accurate data were available. Although the sensitivity of some of these methods to noise has been tested (Bakhtiari-Nejad et 
al 2005, Caddemi and Morassi 2007, Terlaje and Truman 2007, Yang and Sun 2010, Abdo 2012, Boumechra 2017, Greco et al 2019), they were not specifically designed for dealing with noisy data. In contrast, the main aim of this paper is to estimate stiffness using tools developed for dealing with noise and discrete ill-posed problems. For this purpose, the problem of estimating stiffness through measured displacements is linearized using the unit-force theorem. This allows stating the problem as the product of a coefficient matrix and a stiffness vector, as opposed to methods where the stiffness values of each element are embedded in the global stiffness matrix. It is then possible to implement regularization methods, such as L-curve (Hansen 1992, Hansen and O’Leary 1993) and generalized cross-validation (GCV) (Golub et al 1979, Hansen and O'Leary 1993), that reduce the effect of noise to provide a better estimation of the stiffness profile of a beam. Regularization methods introduce a penalty term in the least-squares problem so that overfitting can be prevented. They aim to achieve an equilibrium between minimizing the objective function (i.e., the difference between measured and computed displacements) and obtaining a smoother solution (i.e., a more realistic stiffness profile).

Firstly, the linearized stiffness-displacement system is presented, and regularization techniques are discussed. Both the L-curve approach and GCV are explored as a means to estimate the penalty term in the regularization. Initially, the focus is placed upon the common case when the only available measures are vertical deflections of the structure. Nonetheless, the possibility of working with rotations is explored further on. A discretized FE model of a beam is used to test the regularized approach using theoretical simulations. Random noise is added to values of theoretical displacements as a percentage of the maximum displacement. Secondly, key parameters and limitations of the method are highlighted. Then, the impact of noise level and number of unknowns on the accuracy of the predicted stiffness is discussed for a sample of 
static responses from beams generated with random distributions of stiffness and noise. Finally, the presence of damage and a statically indeterminate beam are tested.

\section{Methodology}

\section{Linearized stiffness-displacement system}

Here the term displacement is employed to denote either deflections (i.e., vertical translations) or rotations. The unit-force theorem allows calculating the deflection at any given point of a beam by considering two loading states: the real one and another with a virtual unit vertical force at that same point where deflection is sought. For each load state, the bending moment diagrams can be computed and the deflection at the point will be equal to the integral, along the whole length of the beam, of the product of the bending moments divided by the flexural stiffness. Contributions of axial, shear and torsional deformations to deflections are assumed to be small and are neglected. In order to compute this integral, the points denoting (i) the presence of a real load, (ii) the presence of the virtual unit force and, (iii) a change in stiffness, must be identified. Then, the integral throughout the entire structure is divided into smaller integral intervals with boundaries defined by every two consecutive points (i), (ii) or (iii). Given that a discretized model is employed, there will be one interval boundary related to points (iii) for each of the end nodes defining the elements that characterize the stiffness distribution throughout the beam. For example, Figure 1 shows a discretized beam formed by 3 segments of equal length distinguished by the stiffness $(E I)_{1},(E I)_{2}$ and $(E I)_{3}$. Two point loads of equal magnitude $P$ acting on points $\mathrm{B}$ and $\mathrm{C}$ simulate a four-point flexural test, commonly used in the determination of the stiffness of a beam. Here, the deflection of point $\mathrm{A}$ is calculated dividing the integral into six parts as per Equation (1). 


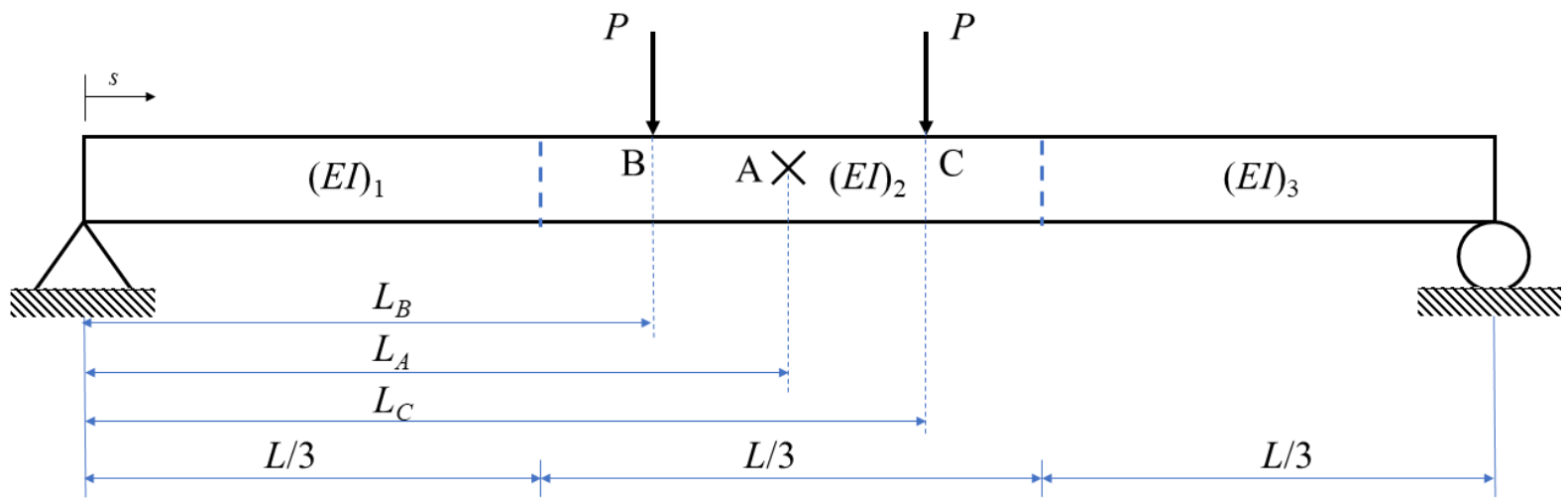

Fig. 1. Four-point flexural test of beam model discretized into three elements

$$
v_{A}=\int_{0}^{L}\left(\frac{M}{E I}\right) M^{u} d s=\int_{0}^{L / 3} \frac{M M^{u}}{(E I)_{1}} d s+\int_{L / 3}^{L_{B}} \frac{M M^{u}}{(E I)_{2}} d s+\int_{L_{B}}^{L_{A}} \frac{M M^{u}}{(E I)_{2}} d s+\int_{L_{A}}^{L_{C}} \frac{M M^{u}}{(E I)_{2}} d s+\int_{L_{C}}^{2 L / 3} \frac{M M^{u}}{(E I)_{2}} d s+\int_{2 L / 3}^{L} \frac{M M^{u}}{(E I)_{3}} d s
$$

where $M$ and $M^{u}$ are the bending moment diagrams due to the applied loads and due to a unit vertical force at the measurement point $\mathrm{A}$, respectively; $(E I)_{i}$ represents the stiffness for the segment $i$ of the beam and, $L_{k}$ and $L_{k+1}$ are the integral boundaries. In Equation (1), the space between the lower $(s=0)$ and upper $(s=L)$ limits of integration is divided into intervals to express the original integral covering the entire beam length as a sum of several integral 'parts' with a known solution. The term 'parts' is used here to refer to the number of addends needed to compute the integral, in contrast to the number of discretized elements of the beam with assumed uniform stiffness. The difference between the number of elements of the beam ( 3 in Equation (1)) and the number of addends of the integral (5 in Equation (1)) derives from the presence of points (i) and (ii). For example, parts two through five of the integral in Equation (1) refer to the same $(E I)_{2}$, but the integral is split into 4 parts given that both $M$ and $M^{u}$ 
distributions present points where the mathematical expression defining the bending moment as a function of the beam location changes. The final goal is to have the bending moment defined by only square, trapezoidal or triangular shapes, which makes the computation of the integral easier. The integrals of the product of bending diagrams $M$ and $M^{u}$ can be computed first, i.e., via the application of tables of virtual work integration (Ghali and Neville 2017), disregarding the value of stiffness, which is assumed to be constant for each integral interval. Then, each product is assigned the stiffness corresponding to the portion of the beam that is related to each interval.

If there were deflections available at $m$ different locations, then there would be a system of $m$ equations as given by Equation (2), that can be built in a similar fashion to Equation (1), but where $M^{u}$ needs to be modified accordingly.

$$
v=A \cdot x \rightarrow\left\{\begin{array}{c}
v_{1} \\
\vdots \\
v_{j-1} \\
v_{j} \\
v_{j+1} \\
\vdots \\
v_{m}
\end{array}\right\}=\left[\begin{array}{ccccc}
a_{1,1} & \ldots & a_{1, i} & \ldots & a_{1, n} \\
\vdots & \ddots & \vdots & \ddots & \vdots \\
a_{j-1,1} & \ldots & a_{j-1, i} & \ldots & a_{j-1, n} \\
a_{j, 1} & \ldots & a_{j, i} & \ldots & a_{j, n} \\
a_{j+1,1} & \ldots & a_{j+1, i} & \ldots & a_{j+1, n} \\
\vdots & \ddots & \vdots & \ddots & \vdots \\
a_{m, 1} & \ldots & a_{m, i} & \ldots & a_{m, n}
\end{array}\right] \cdot\left\{\left(\begin{array}{c}
1 / E I_{1} \\
\vdots \\
1 / E I_{i} \\
\vdots \\
1 / E I_{n}
\end{array}\right)\right\}
$$

where $\mathbf{v}$ is an $m \cdot 1$ vector representing the deflections at different points of the structure, $\mathbf{A}$ is an $m \cdot n$ matrix of coefficients obtained from applying the unit-force theorem and $\mathbf{x}$ is an $n \cdot 1$ vector containing the inverse of the flexural stiffness values. Given that regularization methods are conceived to work in cases where $m>n$, the number of elements needs to be smaller than the number of available measures.

Moreover, the unit-force theorem can be applied to rotations in the same manner. Instead of considering a virtual unit vertical force, it is then necessary to introduce a virtual unit bending 
moment. Thus, the rotation at any given point of the structure can be computed by considering two different bending diagrams, as previously discussed. Ultimately, an equation similar to Equation (2) will be found but with the left-hand side corresponding to a vector $\boldsymbol{\theta}$ of rotations and different terms in the coefficient matrix A. Since these are linear systems of equations, it is possible to combine deflections and rotations so that the problem is reduced to a single system of equations.

\section{Regularization methods}

The basic principle behind these methods is the Tikhonov regularization, which seeks to minimize the function defined in Equation (3):

$$
\Phi_{\lambda}(x)=\left\|A \cdot x-v_{\text {noisy }}\right\|^{2}+\lambda^{2} \cdot\|x\|^{2}
$$

where $\lambda$ is the regularization parameter and $A \cdot x=v$, the linear problem to be solved; furthermore, $\mathbf{v n o i s y}$ is the independent vector of experimental values or measures $\mathbf{v}$ corrupted by noise. If the regularization parameter is assumed to be zero, the methodology applied is simply the same as least-squares. Therefore, Equation (3) seeks to minimize a function composed of two terms: (i) the difference between vnoisy and $\mathbf{v}$, where the latter is calculated using the linear problem; and (ii) a penalty term expressed as the product of the regularization parameter and the vector of unknowns. In this paper, the norm of the vector of unknowns is included in the penalty term as a means of improving the smoothness of the solution. Regularization is applied as a filter to prevent the overfitting of the theoretical displacements to the experimental ones corrupted by noise. This is an approach consistent with Tikhonov regularization. No assumption is made about the physical meaning of the vector of unknowns in the specific problem at hand, and the solution is driven only by the input data. Nonetheless, a different penalty term could be potentially adopted if a priori information about the unknowns is 
assumed. For instance, Ma and Solis (2019) include a penalty term based on an 11 linear trend filter and the prior knowledge of the solution being a piecewise linear function.

Equation (3) can be further developed as shown in Equation (4).

$$
\begin{gathered}
\Phi_{\lambda}(x)=\left(A \cdot x-v_{\text {noisy }}\right)^{t} \cdot\left(A \cdot x-v_{\text {noisy }}\right)+\lambda^{2} \cdot x^{t} \cdot x=x^{t} \cdot A^{t} \cdot A \cdot x-x^{t} \cdot A^{t} \cdot v_{\text {noisy }}- \\
v_{\text {noisy }}^{t} \cdot A \cdot x+v_{\text {noisy }}^{t} \cdot v_{\text {noisy }}+\lambda^{2} \cdot x^{t} \cdot x
\end{gathered}
$$

where a superscript $t$ indicates the transpose of a matrix or vector. It should be noted that the second and third terms in the second part of Equation (4) are equal and can be added together. Equation (5) represents the derivative of Equation (4) with respect to the vector of unknowns $\mathbf{x}$. The derivative is made equal to zero to obtain the minimum of the function represented in Equation (3).

$$
\frac{d \Phi_{\lambda}(x)}{d x}=2 \cdot A^{t} \cdot A \cdot x-2 \cdot A^{t} \cdot v_{n o i s y}+2 \cdot \lambda^{2} \cdot x=0
$$

Then, Equation (5) can be rearranged in the form of Equation (6) so that its structure resembles the original linear problem.

$$
\left(A^{t} \cdot A+\lambda^{2} \cdot I\right) \cdot x=A^{t} \cdot v_{\text {noisy }}
$$

where $\mathbf{I}$ is the identity matrix. Finally, by isolating the vector of unknowns, the closed-form solution provided by Equation (7) is obtained.

$$
x_{\lambda}=\left(A^{t} \cdot A+\lambda^{2} \cdot I\right)^{-1} \cdot A^{t} \cdot v_{\text {noisy }}
$$

For a given set of noisy measures, the solution to Equation (7) is not unique, but it depends on the selected value for the regularization parameter $\lambda$. A series of techniques are needed for selecting the value of the regularization parameter, and L-curve and GCV are amongst the most popular techniques. In the case of the L-curve, two parameters need to be calculated for each 
value of $\lambda$ : the residual norm $\left(\right.$ res $\left._{\text {norm }}\right)$ and the solution norm $\left(\operatorname{sol}_{n o r m}\right)$ given by Equations (8) and (9), respectively.

$$
\begin{gathered}
\operatorname{res}_{\text {norm }}=\left\|A \cdot x_{\lambda}-v_{\text {noisy }}\right\| \\
\text { sol }_{\text {norm }}=\left\|x_{\lambda}\right\|
\end{gathered}
$$

Then, both norms are calculated for each potential value of $\lambda$ and plotted, with the residual norm in the horizontal axis. In ideal circumstances, the graph is expected to have an $\mathrm{L}$ shape with a vertical part in the left-hand side and a flat part in the right-hand side. The optimal value of the regularization parameter would correspond to the point of maximum curvature when the transition from flat to vertical happens. Often, the graphic is plotted using logarithmic scales to better appreciate the point of maximum curvature. It must be noted that in an ill-posed problem, the transition between both parts of the curve may not be as sharp as in an ideal case, and the point of maximum curvature may not always be easy to identify.

In the case of GCV, the available data are divided into folds of the same size. Then, one of the folds is left out and the model is trained using the rest of them; that is, Equation (7) is solved not for all the data but for a portion of them. Once a solution has been obtained, it is used to solve the linear equation and predict the remaining observations. The process is repeated leaving out one different fold each time until all folds have been tested. At each step, the square error between the predicted observations and the data left out is computed; finally, the crossvalidation error can be defined as the mean of all the errors. Selecting the size of the folds depends on the problem at hand and the number of available observations. If only one datum is left out at a time, this approach is called leave-one-out cross-validation. The latter can be time-consuming in the case of large sets of data, but it will be adopted here given the relatively small number of available measures. The GCV error is then computed as: 


$$
G C V=\frac{1}{m} \cdot \sum_{j=1}^{m}\left(\frac{v_{\text {noisy }}^{i}\left(A \cdot x_{\lambda}\right)^{i}}{1-\operatorname{trace}\left(A \cdot\left(A^{t} \cdot A+\lambda^{2} \cdot I\right)^{-1} \cdot A^{t}\right) / m}\right)^{2}
$$

where $m$ is the total number of measures and trace indicates the sum of the elements of the main diagonal of the matrix $\left(A \cdot\left(A^{t} \cdot A+\lambda^{2} \cdot I\right)^{-1} \cdot A^{t}\right)$. The optimal regularization parameter can be chosen then as the one that yields a minimal GCV error. As a limitation, when correlated noise is expected, cross-validation can result in an underestimation of the regularization parameter.

\section{Implementation of regularization methods}

A FE model of a simply supported beam is employed to test the proposed methodology. The total span between supports is $2.4 \mathrm{~m}$, and the loading conditions are defined by two concentrated loads $(2.5 \mathrm{kN}$ each) placed at $1.06 \mathrm{~m}$ from each support. The FE model is discretized into 10 beam elements/m (24 elements in total, i.e., $n=24)$. Normal probability distributions are considered for the stiffness of each element. The stiffness profile is generated randomly assuming spatial correlation between the beam elements. The varying stiffness profile has been chosen to simulate small variations of stiffness that can be expected in a reinforced concrete beam of constant cross-section, not only due to the inherent variability of concrete but also to corrosion. The correlation function (Hajializadeh et al 2016) is defined by Equation (11). As a result, the obtained stiffness profiles are smooth, with realistic variations between consecutive elements.

$$
\rho=\rho_{0}+\left(1-\rho_{0}\right) \cdot e^{-\left(\frac{\tau_{x}}{d_{x}}\right)^{2}}
$$


where $\tau_{x}$ represents the distance between element centroids, $\rho_{0}$ is a constant correlation component and $d_{x}$ is related to the scale of fluctuation, i.e., it controls the distance at which correlation becomes irrelevant.

\section{Motivation}

In a preliminary analysis, a simulation is conducted in a scenario where the number of measures is considerably higher than the number of unknowns. Deflections are assumed to be measured every $1 \mathrm{~cm}$; hence, a total of 239 measures are available $(m=239)$. The theoretical deflection of the beam is obtained for a distribution of stiffness based on a mean value of $250 \mathrm{kNm}^{2}$ with a standard deviation of $12.5 \mathrm{kNm}^{2}$ (5\% of the mean stiffness), and correlation parameters $d_{x}=$ $0.4 \mathrm{~m}$ and $\rho_{0}=0.2$. Noise is assumed to follow a normal distribution with zero mean and a $2 \%$ of the maximum deflection as standard deviation, that is randomly added to the theoretical deflection. This level of noise can represent a significant percentage with respect to the exact value in locations far from the maximum deflection. Figure 2 shows the exact values and generated noisy measures for each measurement position, $p$, in the beam. 


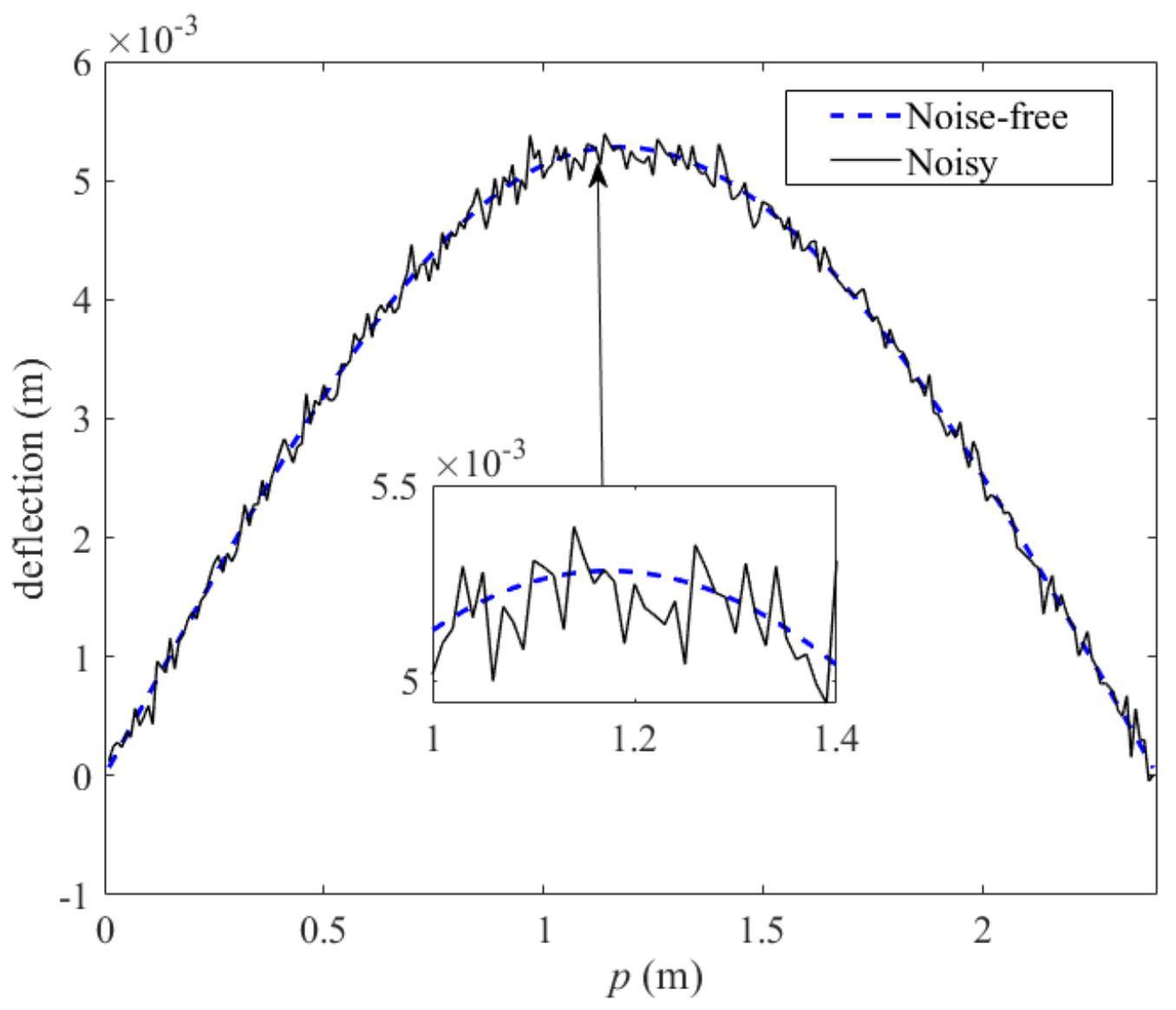

Fig. 2. Noisy and noise-free measurements

For comparison purposes, Figure 3 shows the stiffness profile obtained from applying a leastsquares approach (i.e., without regularization or $\lambda=0$ ) to best fit the noisy data in Figure 2. Negative values of stiffness can be seen in the predicted profile. These are obviously unrealistic values, with mathematical significance (best-fit to the simulated measures), but no physical meaning, since negative flexural stiffness is not possible. This figure serves to illustrate the limitations of least-squares in cases where relatively high noise is present in the measures. 


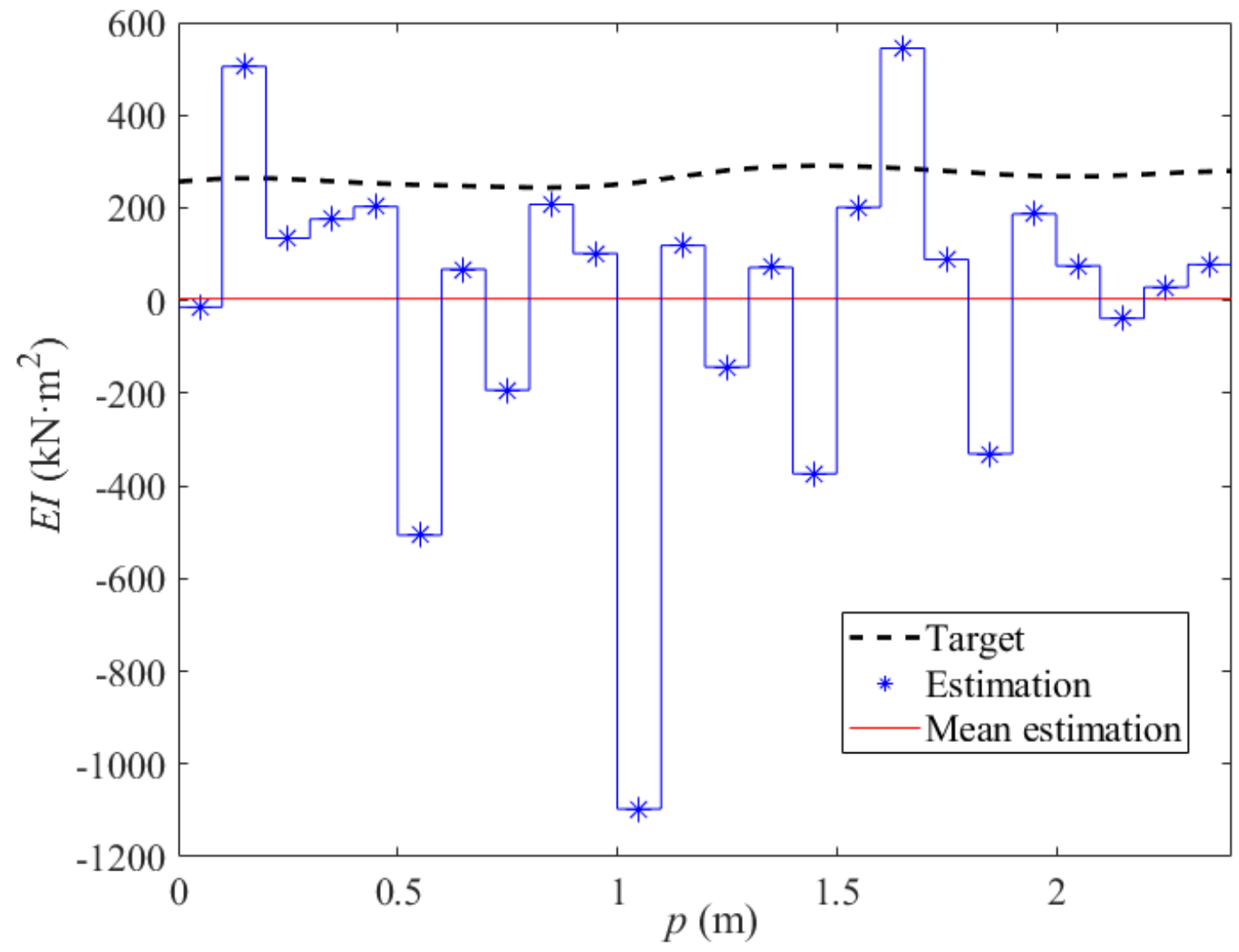

Fig. 3. Least-squares estimation of stiffness profile

The target stiffness profile in Figure 3 is generated from a multivariate normal distribution, created using Equation (11) so that spatial correlation is taken into account. It should be noticed from the figure that this profile is smooth but not constant. The presence of noise, although apparently low, especially for measures close to mid-span, prevents the least-squares approach from establishing a valid solution with only positive values of stiffness. These conclusions can be extended to most of the predictions performed applying a least-squares approach $(\lambda=0)$ to the noisy simulated data employed in this paper. Clearly, a regularization parameter needs to be considered to reduce the influence of noise on accuracy.

Recommendations on the selection of the regularization tool and parameter

Implementation of the L-curve method 
The L-curve method is implemented varying $\lambda$ in a range from $10^{-3}$ to $10^{3}$. The L-curve, corresponding to the same data employed for the stiffness estimation of Figure 3, is plotted in Figure 4 and a typical L-shape can be noticed. On the one hand, the vertical portion of the curve to the left of the figure corresponds to solutions of the stiffness profile that minimize the difference in deflections (Equation (8)). For these cases, however, the solution norm (Equation (9)) tends to increase considerably. On the other hand, the flat part of the curve to the right of the figure represents smoother solutions of the stiffness profile with an increasing error in the fit of the calculated to the measured deflections (i.e., higher values of Equation (8)). The selected point of maximum curvature, marked with a cross, represents the balance between these two components of the error. Nonetheless, the computation of the maximum curvature point can prove to be troublesome. Here, the curvature is approximated considering three points of the curve. The transition between the two parts of the curves in Figure 4 is quite sharp, but this is not always the case. Thus, the difficulty to accurately select the point of maximum curvature becomes the main limitation in the use of the L-curve approach. 


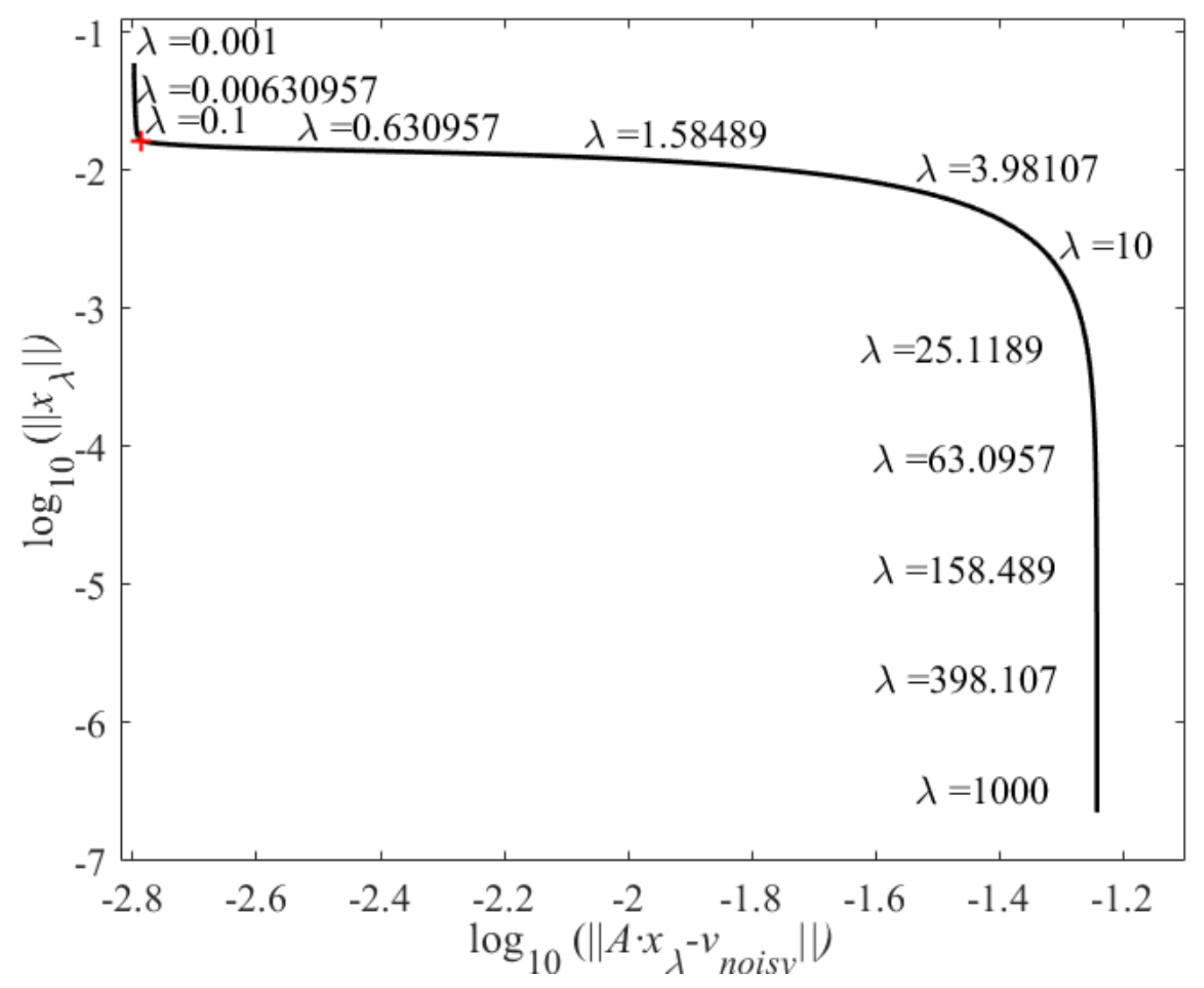

Fig. 4. L-curve corresponding to noisy data from Figure 2

In a practical situation, the actual value of the stiffness will typically be unknown, but in a numerical simulation, it is possible to determine the prediction error of stiffness for each value of the regularization parameter to assess the accuracy of the regularization method. The optimal $\lambda$ would be the one that minimizes the prediction error, which is defined here as:

$$
e_{i}(\%)=100 \cdot \frac{\sqrt{\left(E I_{\lambda, i}-E I_{i}\right)^{2}}}{E I_{i}}
$$

where $e_{i}$ refers to the prediction error for element $i ; E I_{\lambda, i}$ represents the prediction for the stiffness of element $i$ with a given value of the regularization parameter $(\lambda)$; and $E I_{i}$ corresponds to the actual stiffness value for the same element. 
Figure 5 shows the prediction error for all elements in the surroundings of the maximum curvature of the L-curve, between $10^{-3}$ and $10^{0}$. While the prediction errors for the two elements closer to each support are shown using four thin solid lines, the prediction errors for the inner elements are shown using dashed lines. The thick black line corresponds to the average prediction error for all elements.

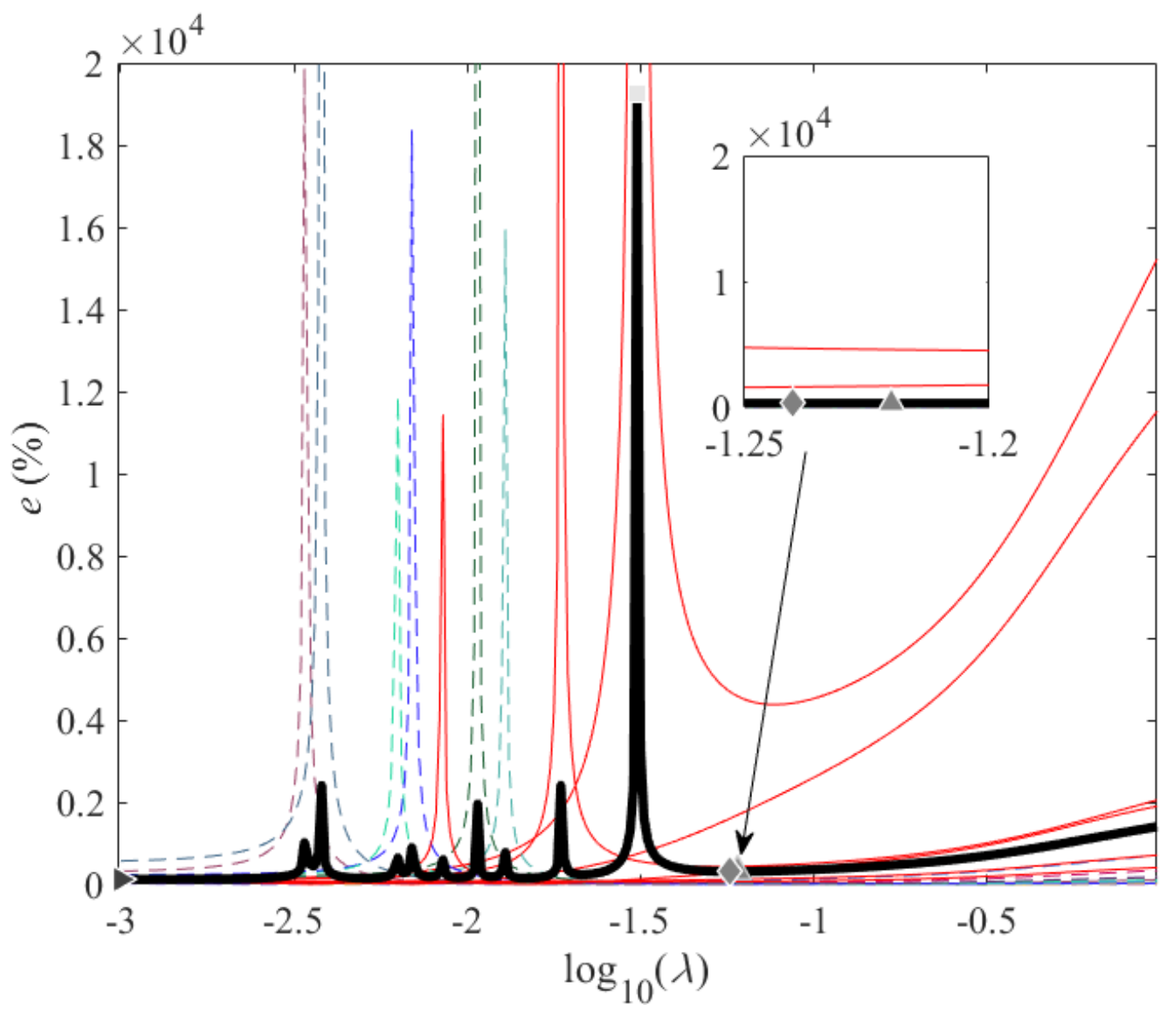

Fig. 5. Average prediction error vs $\lambda$

It can be seen that for small values of the regularization parameter (left side of Figure 5); there are spikes in the prediction error that occur at a different $\lambda$ for each element. It is worth to highlight that each spike represents the transition of an element from a negative stiffness value to a positive one. Nine spikes of different magnitude can be seen in Figure 5., i.e. the spike at $\lambda=10^{-2.42}$ corresponds to beam element number 11 between 1 and $1.1 \mathrm{~m}$ from the left support. 
Once this value of the regularization parameter is overpassed (i.e., to the right of the spike in the figure), no negative stiffness prediction will be found for this element. The last spike (element number 1 between 0 and $0.1 \mathrm{~m}$ from the left support) for $\lambda=10^{-1.51}$ is followed by a sudden decrease and, finally, a gradual increase in the prediction error. In the figure, the point of minimal average prediction error is marked with an arrowhead (left bottom corner of the figure); in this case, it matches the lowest value of the range considered for $\lambda\left(\lambda_{1}=10^{-3}\right)$. However, given that it precedes all spikes in the figure, the associated stiffness profile contains negative stiffness values, similarly to the least-squares solution in Figure 3. The maximum value of $\lambda$ for which negative stiffness values are still present in the solution is marked in Figure 5 using a square. The latter corresponds to the rightmost spike in the average prediction error, which takes place for $\lambda=10^{-1.51}$. It is found that, beyond that threshold value of the regularization parameter, $\lambda_{t h}=10^{-1.51}$, no stiffness profile contains negative values. This behaviour is consistent for all simulations conducted.

From the findings above, the optimal regularization parameter can be defined as the one that provides the minimum average prediction error while being greater than $\lambda_{t h}$. In this case, this value $\left(\lambda_{2}\right)$, marked as a triangle in Figure 5 , is $10^{-1.22}$ and leads to an average prediction error for all elements of $310.25 \%$. This value is high but expected due to the unreliable estimations of stiffness for the elements closest to the supports, which barely contribute to the overall deflection of the structure. Figure 5 shows how the prediction error (thin solid lines) for the four elements near the supports is much greater than the rest and it diverges as the regularization parameter increases towards the right side of the figure. Thus, it seems reasonable to analyse the prediction error considering only the 20 inner elements of the beam, i.e., removing the two elements closest to each support from the error calculations. In this case, the minimum prediction error corresponds to $\lambda_{3}=10^{-1.24}$ (marked with a diamond both in Figure 5), for which the error is just $22.69 \%$. If instead of two elements closer to the support, three elements were 
excluded at each beam end, then, the error would drop from $22.69 \%$ to $13.81 \%$. Similarly, if the last four elements at each end of the beam were excluded (the remaining elements still cover $1.6 \mathrm{~m}$, i.e., two-thirds of the length of the beam), the average prediction error is found to be $10.94 \%$. In comparison, a least-squares approach $(\lambda=0)$ as employed in Figure 3 , leads to average prediction errors of $114.89 \%, 119.92 \%, 124.18 \%$ and $133.19 \%$ when considering the 24 elements, the 20 inner elements, the 18 inner elements and the 16 inner elements respectively. More significantly, the least-squares stiffness profile has no physical meaning since it contains negative stiffness values.

In summary, four values of the regularization parameter can be identified in each simulation:

- $\quad \lambda_{1}$ corresponding to the minimum average error prediction for all elements;

- $\quad \lambda_{t h}$ corresponding to the last solution that contains negative stiffness values;

- $\quad \lambda_{2}$ corresponding to the minimum average prediction error beyond $\lambda_{t h}$, and;

- $\quad \lambda_{3}$ corresponding to the minimum average prediction error for the inner elements.

Taking these values of $\lambda$ into account, the solution provided by the L-curve can be analysed in terms of how close it is to $\lambda_{2}$ and $\lambda_{3}$. Although these values will not be known a priori in a real scenario, the theoretical simulations employed here are useful in assessing the best methodology to estimate $\lambda$. A zoom in on the point of maximum curvature of the L-curve around $\lambda=0.1$ can be visualized in Figure 6 . 


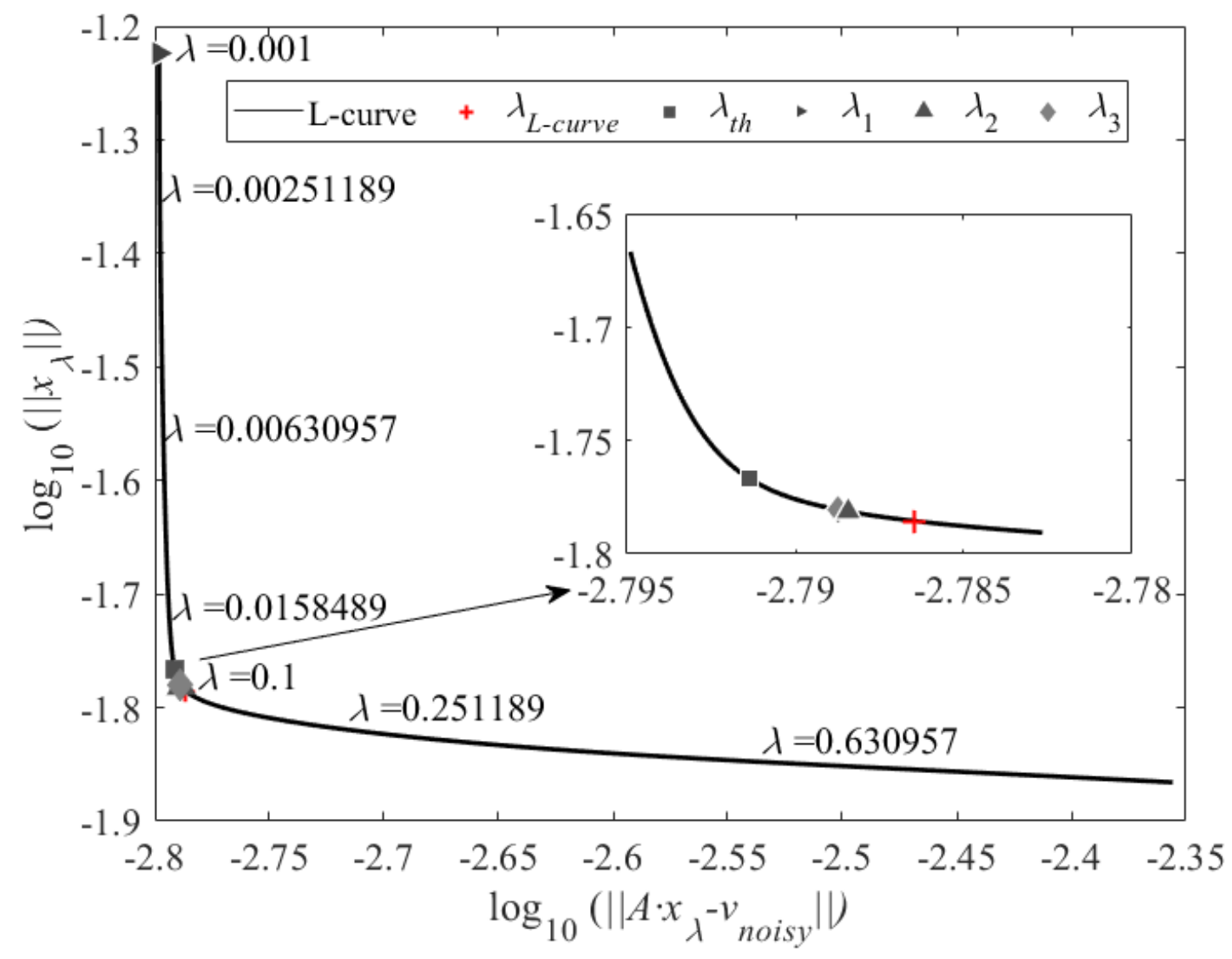

Fig. 6. Detailed L-curve

The aforementioned key values of the regularization parameter are shown in the figure using the same scheme of markers; arrowhead, square, triangle, diamond and cross for $\lambda_{1}, \lambda_{t h}, \lambda_{2}, \lambda_{3}$ and $\lambda_{\text {L-curve }}$ respectively. $\lambda_{1}$ clearly corresponds to the vertical part of the curve while the rest of them are close to the maximum curvature point. A zoom into this region denotes that the selected value for the L-curve $\left(\lambda_{\text {-curve }}=10^{-1.12}\right)$ is close to $\lambda_{2}$ and $\lambda_{3}$. It is difficult to actually state where the point of maximum curvature (cross for $\lambda_{\text {-curve }}$ ) lies exactly. This calculation of $\lambda_{\text {-curve }}$ is very sensitive to the number of points used to compute the curvature. Nonetheless, the average prediction error for the proposed regularization parameter does not vary significantly ( $23.6 \%$ for inner elements using $\lambda_{\text {-curve }}=10^{-1.12}$ ) from the minimum possible error ( $22.69 \%$ for inner elements using $\lambda_{3}=10^{-1.24}$ ) found based on a knowledge of the true stiffness. 


\section{Implementation of GCV}

GCV is an alternative method to the L-curve for finding the ideal $\lambda$. Given the limited number of measures that are typically available in a practical lab or field test, a leave-one-out approach is adopted here as it will not be computationally too expensive. The graphic corresponding to the GCV error is shown in Figure 7. Here, the minimum GCV error is found at $\lambda_{G C V}=10^{-1.12}$, the same value obtained for the L-curve. Key values of $\lambda$, as previously defined, are marked in the figure using arrowhead $\left(\lambda_{1}\right)$, square $\left(\lambda_{t h}\right)$, triangle $\left(\lambda_{2}\right)$ and diamond $\left(\lambda_{3}\right)$ markers. In this case, the proposed regularization parameter obtained using both methods, L-curve and GCV, matches. However, for higher levels of noise or smaller ratios of measurement points over the number of discretized elements, this is not always the case.

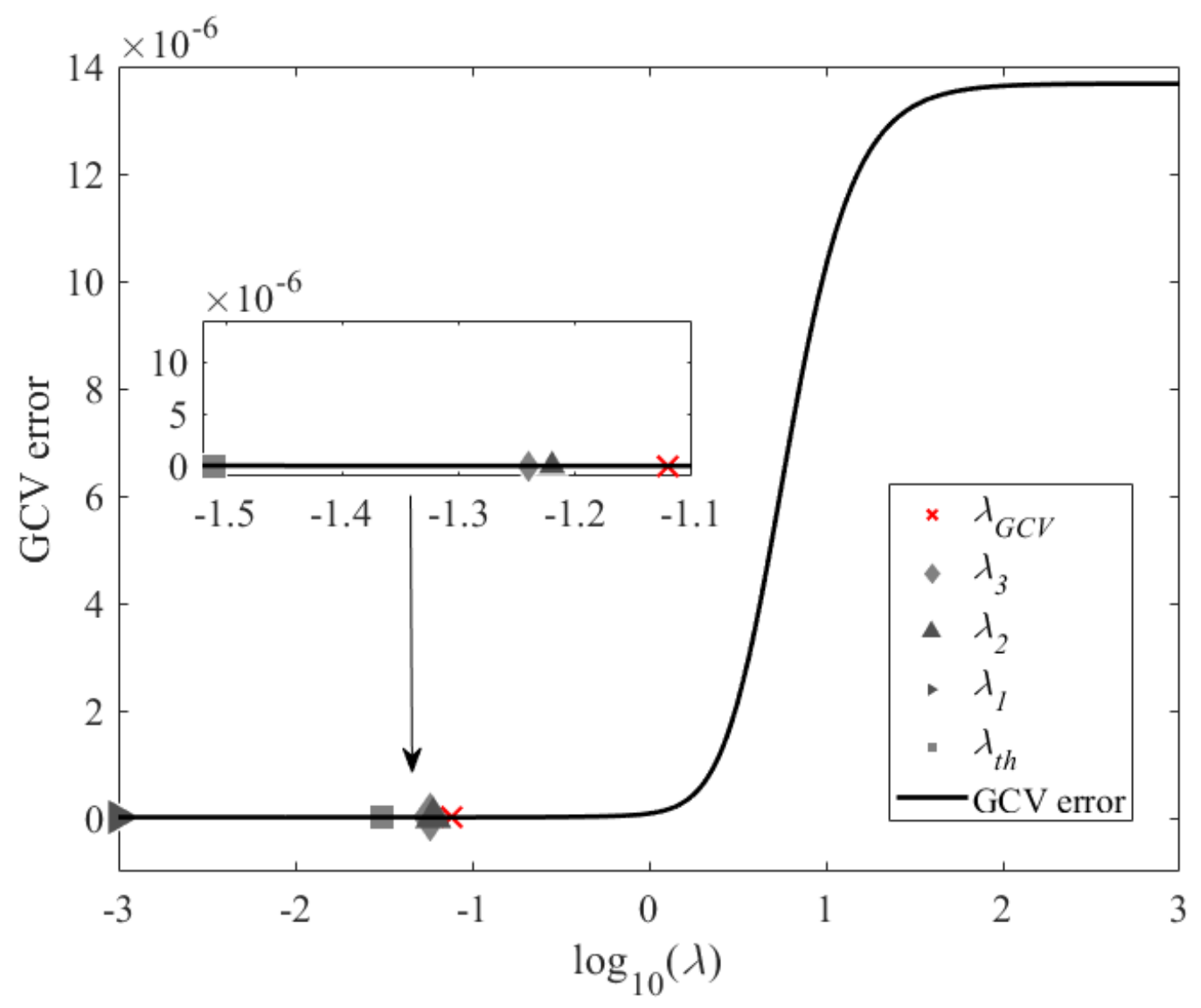

Fig. 7. GCV error vs $\lambda$ for noisy data from Figure 2 
Although the left part of Figure 7 is almost horizontal, a minimum (marked as a cross in the figure) can be identified. In an ideal case, the left end of the curve would show a slight trend upwards that cannot be visualized here. Eventually, the minimum can even be located far apart from the left of the curve for some cases of correlated noise. It is then when the GCV parameter chosen according to this minimum could significantly differ from the optimal value.

Figure 8 shows the stiffness profile estimated using the regularization parameter value proposed by both the L-curve and generalized cross-validation, i.e., $\lambda=10^{-1.12}$.

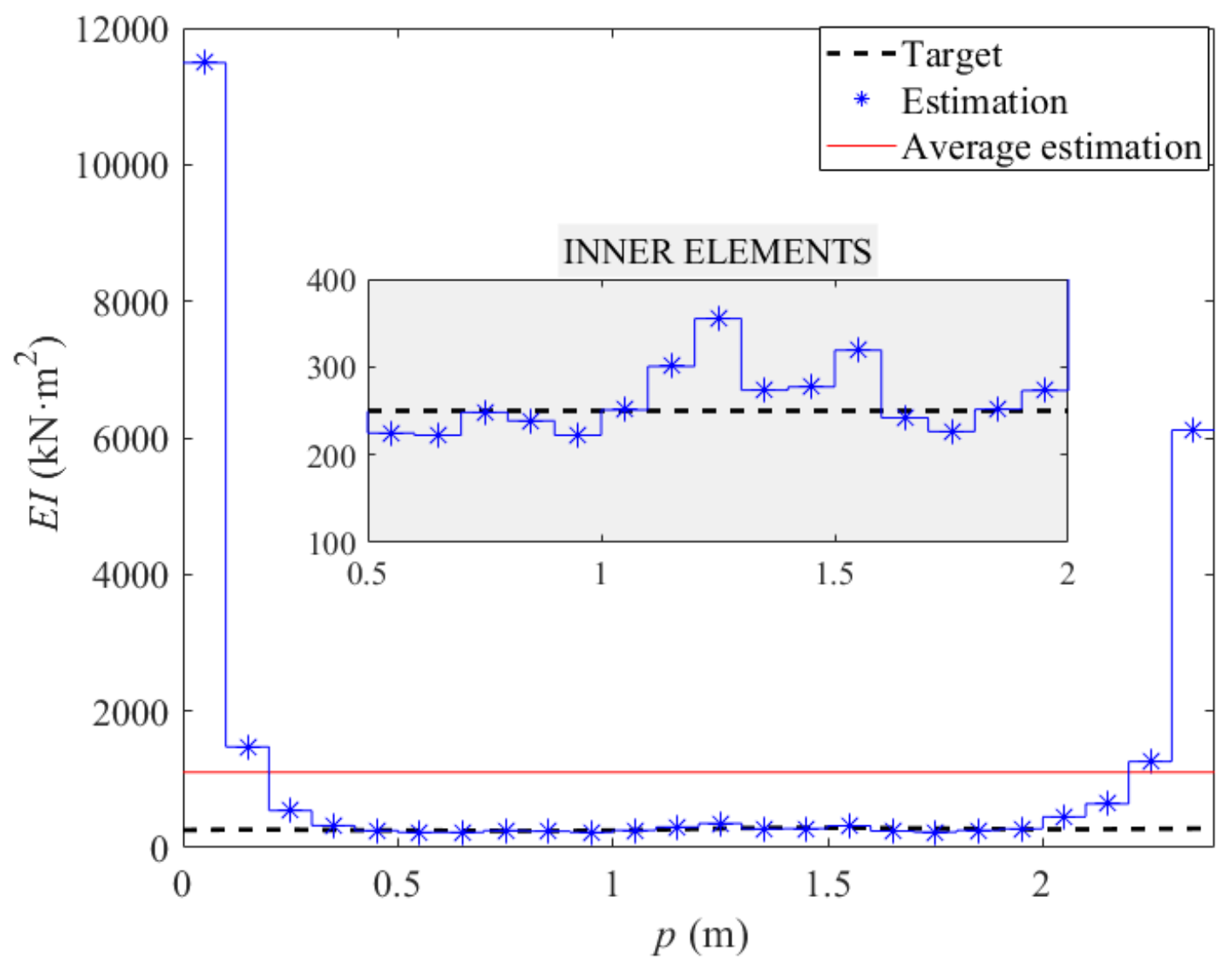

Fig. 8. Stiffness profile, $\lambda=10^{-1.12}$

As it is usually the case, the prediction error for the elements closest to the supports is inaccurate, but the prediction of the stiffness profile of the inner elements in Figure 8 using regularization clearly outperforms the prediction using least-squares in Figure 3. Finally, 
Figure 9 compares the deflections that would result from using the stiffness profiles provided by the regularization methods to the least-squares solution and to the noisy simulated measures.

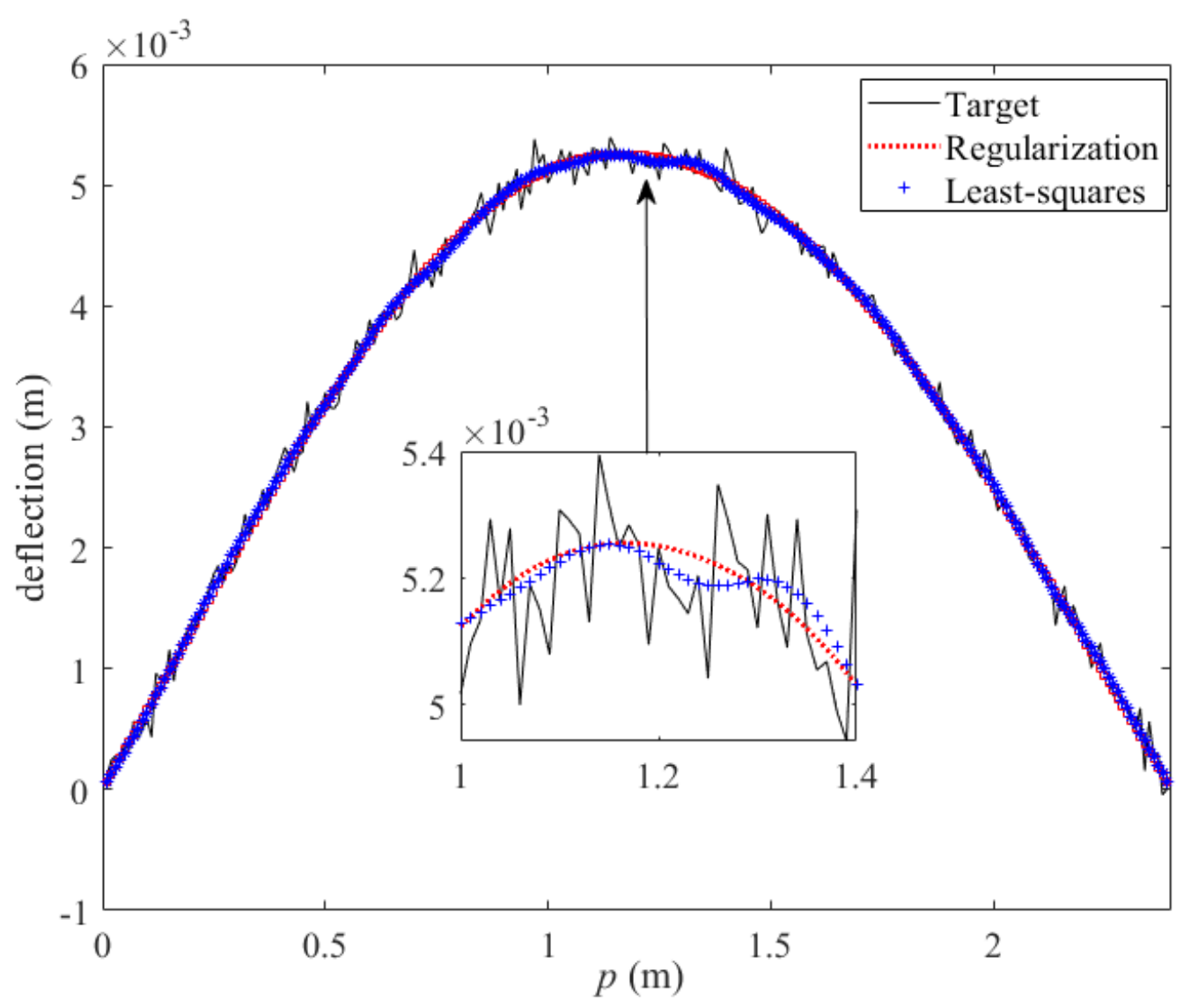

Fig. 9. Predicted deflections versus measurement location in the beam

It can be seen how the least-squares approach results in deflections that adapt better to the noise but provide a more irregular shape, far from what it is expected from a beam with a quasiuniformly distributed stiffness. On the other hand, the deflections obtained from the regularization methods, while adapting to the noisy measures, maintain the typical deflection profile of a simply supported beam of homogenous stiffness.

Testing using deflections and rotations with different levels of noise and number of unknowns in a statically determinate beam 
Section 3 has employed a generic example to illustrate how the regularization methods work and to discuss their limitations and challenges when selecting the optimal regularization parameter. One stiffness profile consisting of 24 elements is randomly generated by means of a multivariate normal distribution with mean of $250 \mathrm{kNm}^{2}$ and standard deviation of $5 \mathrm{kNm}^{2}$, for which correlation values are defined according to Equation (11) with $\rho_{0}=0.2$ and $d_{x}=1.2$ m. Three different levels of noise are considered: $0.5 \%, 1 \%$ and $2 \%$. Noise is added to the displacements associated with the theoretical stiffness profile to generate 5000 sets of displacements for each noise level. This set of simulations aims to analyse the performance of the regularization methods with a more practical number of measures, i.e., taken every $10 \mathrm{~cm}$, compared to Section 3 with measures every $1 \mathrm{~cm}$. Therefore, deflections are obtained at 23 locations $(m=23)$, and rotations at 24 locations $(m=24)$. In both cases, locations where the signal equals zero (mid-span for rotations and supports for deflections) are disregarded. As for the number of parameters to be estimated, firstly, only one unknown is considered (i.e., $n=1$ ) to test the ability of the methodology in predicting the average stiffness of the beam. Secondly, two unknows are predicted, i.e., one stiffness value assigned to each half of the beam $(n=2)$. Furthermore, the number of unknowns is sequentially increased. In total, seven different scenarios with $n=1,2,3,4,6,8$ and 12 are considered.

As previously discussed, the resulting noise can eventually result in sub-optimal choices of the regularization parameter, given the aforementioned limitations of the L-curve (inaccuracy in identifying the point of maximum curvature) and GCV (decreasing or flat curve resulting in an estimation of a value of the regularization parameter far too low). In order to address these limitations, the following criteria are applied:

- If $\lambda_{L \text {-curve }} \leq \lambda_{t h}$, the simulation is classified as "L-curve fail".

- If $\lambda_{G C V} \leq \lambda_{\text {th }}$, the simulation is classified as "GCV fail". 
- If $\lambda_{L-c u r v e} \leq \lambda_{\text {th }}$ and $\lambda_{G C V} \leq \lambda_{\text {th }}$, the two methods are unable to select an optimal parameter, and the simulation is labelled as "Fail".

Therefore, the simulations where the selected regularization parameter results in a stiffness profile with negative values are considered to have failed since such a stiffness profile is not physically possible. Failure is often the result of specific noisy displacements that lead to conditions in the L-curve and GCV plots that make the selection of the optimal parameter unfeasible.

The first necessary step is to identify the failed simulations in order to exclude them from the statistical analysis of the results. The number of failed simulations also provides an idea of the robustness of each method (L-curve, GCV), and it is found that overall GCV is far more reliable than the L-curve. Three general remarks can be made: (i) for $n \leq 4$, no failed simulation is found for any level of noise or type of input (rotations/deflections); (ii) in the case of rotations, this limit increases to $n=8$; (iii) for $0.5 \%$ noise, no failed simulations are obtained when using rotations as inputs. In the scenarios where failed simulations appear (high number of unknowns $n$ and $1 \%$ and $2 \%$ noise), the criterion most commonly met is that of "L-curve fail". In the case of rotations and $2 \%$ noise, L-curve fails in $17 \%$ of simulations whereas the number of simulations labelled as "GCV fail" is only $1 \%$ for $n=12$.

On the other hand, the performance of both methods declines when using deflections as inputs. For instance, in the case of 12 unknowns, "Fail" represents $0.52 \%, 1.32 \%$ and $2.14 \%$ of all simulations using deflections for each increasing level of noise. Moreover, even if simulations classified as "Fail" and "GCV fail" (less than $1 \%$ for any value of $n$ ) are not significant; the number of "L-curve fail" simulations is considerable, up to $30 \%$ for the case of 12 unknowns and $0.5 \%$ noise. Hence, it is clear that GCV is more reliable than the L-curve and results presented here will focus on the former. The tendency of the L-curve to produce failed 
simulations can be related to the difficulty in localizing the point of maximum curvature and to a systematic selection of lower values of the regularization parameter. If the values of the regularization parameter obtained from both methods are compared for all scenarios considered, GCV is always found to yield a larger value of $\lambda$ than the L-curve. Thus, the Lcurve favours solutions that are smoother while GCV tends to produce solutions that minimize the error in displacement.

\section{Results using rotations}

The performance of the GCV-based algorithm using rotations is presented in Figure 10 for the lowest level of noise $(0.5 \%)$. In this figure, the first and last rows indicate the length (in $\mathrm{m}$ ) of the beam portion for which stiffness is being predicted. On the other hand, the inner rows of Figure 10 give the mean $(\mu)$ and standard deviation $(\sigma)$ of the percentage error in the prediction for the average value of the represented length. For example, if 8 unknowns were considered $(n=8)$, the third unknown would represent the segment of the total beam between 0.6 and 0.9 $\mathrm{m}$. Then, the average value of the true stiffness profile over that length is computed to calculate the prediction error shown in the figure by comparing it to the predicted stiffness.

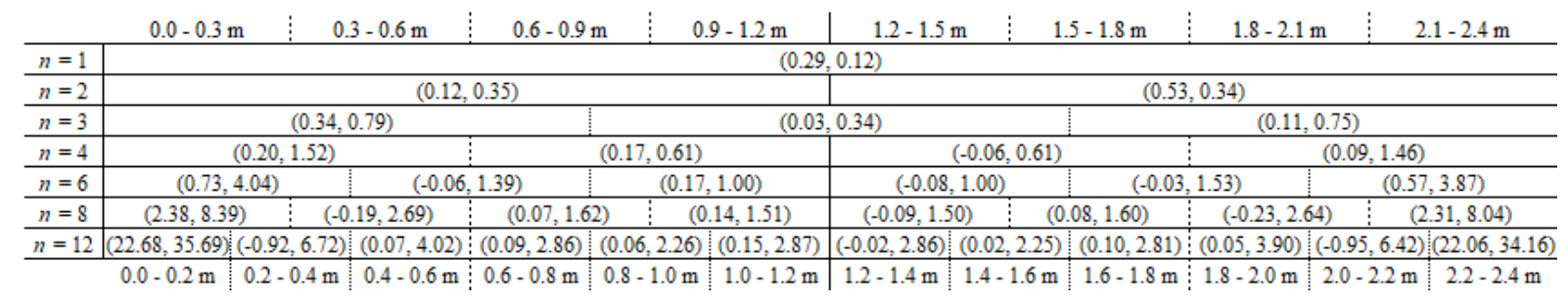

Fig. 10. Statistical parameters $(\mu, \sigma)$ of percentage error in stiffness using rotations and $0.5 \%$ noise

It can be seen that the prediction error between 0.2 and $2.2 \mathrm{~m}$ is in all cases below $1 \%$ for any number of unknowns considered. However, degradation on the reliability of the prediction is reflected in the standard deviation, which systematically increases when a higher number of 
unknowns is considered. It is also important to note that the standard deviation shows larger values for the segments of the beam located further away from the centre of the beam. For 12 unknowns ( $n=12$ in Figure 10), the prediction for segments of the beam near the supports $(p$ $=0-0.2 \mathrm{~m}$ and $p=2.2-2.4 \mathrm{~m}$ ) can be noticed to be highly unreliable. For this reason, GCV results associated with this fine discretization are omitted from the predictions shown in Figures 11 and 12 for the $1 \%$ and $2 \%$ levels of noise respectively.

The continuous lines in Figures 11 to 15 represent the target stiffness profile for each case, while the dashed lines correspond to the $1 \%$ and $5 \%$ error with respect to that profile. The mean stiffness prediction is marked with a point placed at the centre of the length represented by the unknown. Two additional points are added for each average prediction, corresponding to the pair of values $(\mu-\sigma, \mu+\sigma)$, i.e., mean \pm standard deviation. In Figure 11, the mean stiffness prediction falls within the $5 \%$ range for all cases but the two elements closest to the supports in the case of $n=8$. In particular, the mean stiffness prediction for inner elements $(p=0.4-2$ m) is within $1 \%$ for all number of unknowns considered. On the other hand, mean stiffness predictions for outer elements $(p=0-0.4 \mathrm{~m}, 2-2.4 \mathrm{~m})$ are in the range of $2.3 \%$ and $9.9 \%$ for $n$ $=6$ and $n=8$ respectively. If the confidence interval $(\mu-\sigma, \mu+\sigma)$ is analysed rather than just the mean value, for $n \leq 4$, there is a probability of at least $67 \%$ for the mean stiffness prediction to be within the $5 \%$ range, i.e., all three markers in the same vertical line are within the two dashed lines corresponding to $5 \%$ error. Larger standard deviations are found as the number of unknowns increases $(n=6,8)$ and the analysed element gets closer to the support. 


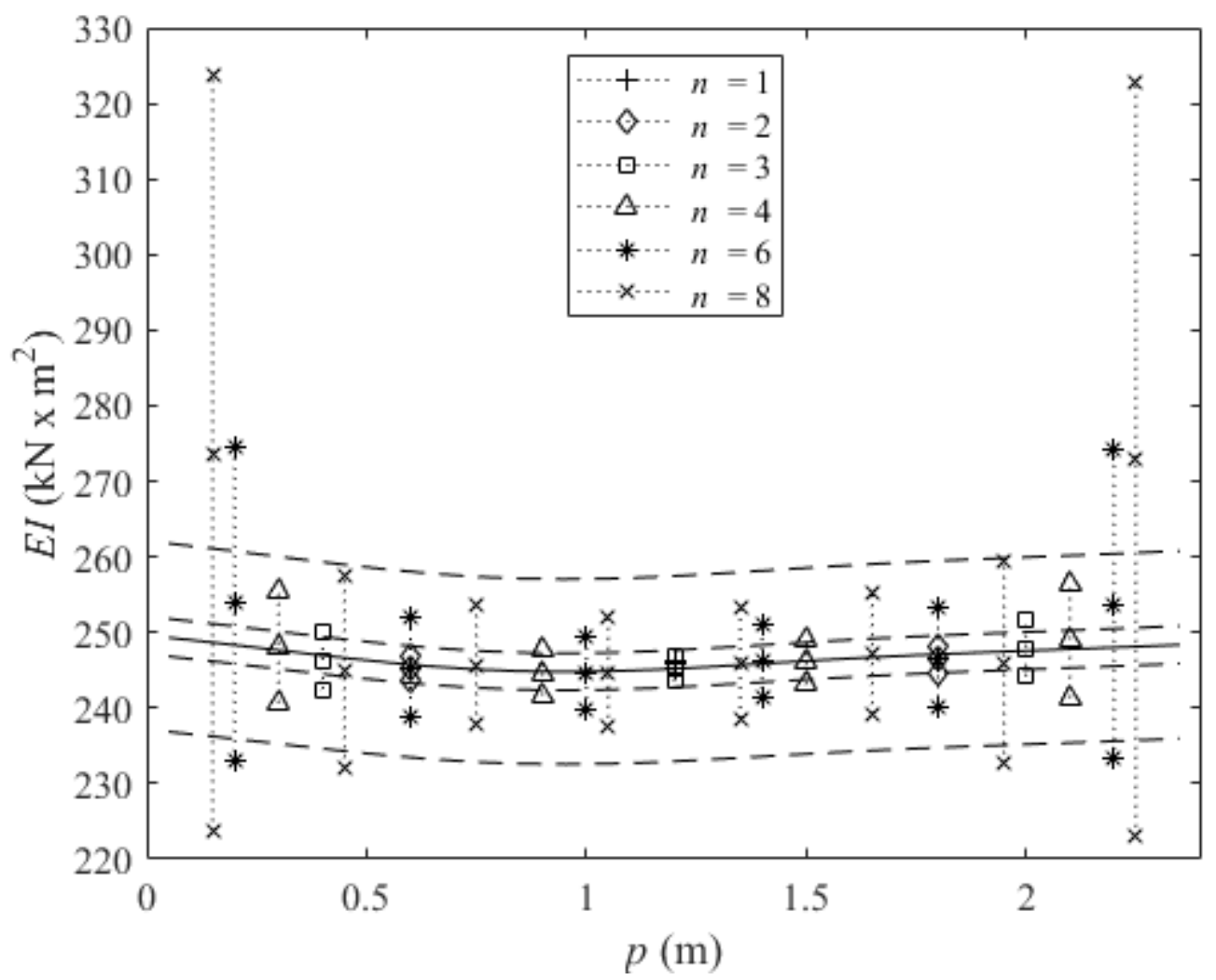

Fig. 11. Stiffness prediction using GCV, rotations and $1 \%$ noise

Figure 12 follows the same pattern as Figure 11 but for the case of $2 \%$ noise. Standard deviations for $2 \%$ noise are generally larger than for $1 \%$. Unlike Figure 11 , for $n=6$ and the element closest to the support, the mean stiffness prediction falls now outside of the $5 \%$ range. Nevertheless, for any element and $n \leq 4$, as well as for $n=6,8$ and any elements but the closest to the support, the mean stiffness prediction still remains within the $5 \%$ range. 


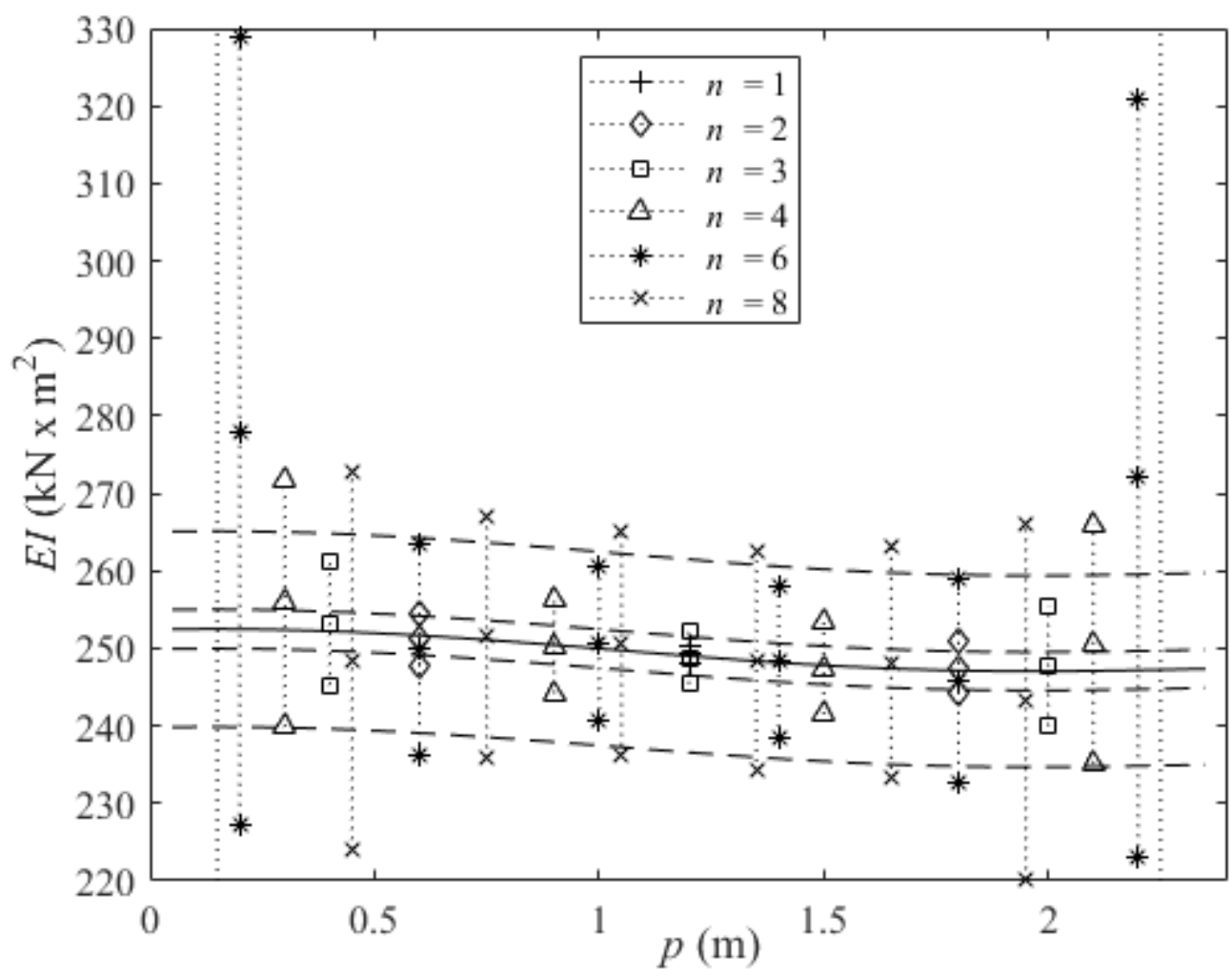

Fig. 12. Stiffness prediction using GCV, rotations and $2 \%$ noise

\section{Results using deflections}

Results obtained by using deflections as inputs, although successful, are less promising than using rotations. As it was the case with rotations, a very high level of discretization $(n=12)$ leads to an unrealistic prediction of the stiffness profiles, thus focus is placed upon a discretization with $n \leq 8$. Figure 13 shows the results for deflections and $0.5 \%$ noise arranged similarly to Figures 11 and 12. In Figure 13, the mean stiffness predictions for $n \leq 4$ remain within the $1 \%$ error range for the $0.5 \%$ level of noise, apart from the closest element to the support in the left-hand side and $n=4$. However, standard deviations are higher in comparison with rotations for a similar level of noise (Figure 10). When considering a higher number of unknowns $(n=6,8)$, the mean error is located in the 1-2\% range, except for the closest element to the support if $n=6$, or the two elements closest to the support if $n=8$. Mean errors for the 
stiffness of elements next to the supports are much higher than those obtained for rotations with a similar level of noise. Standard deviation associated with the predictions using deflections is also larger than the equivalent predictions using rotations regardless of the element under consideration.

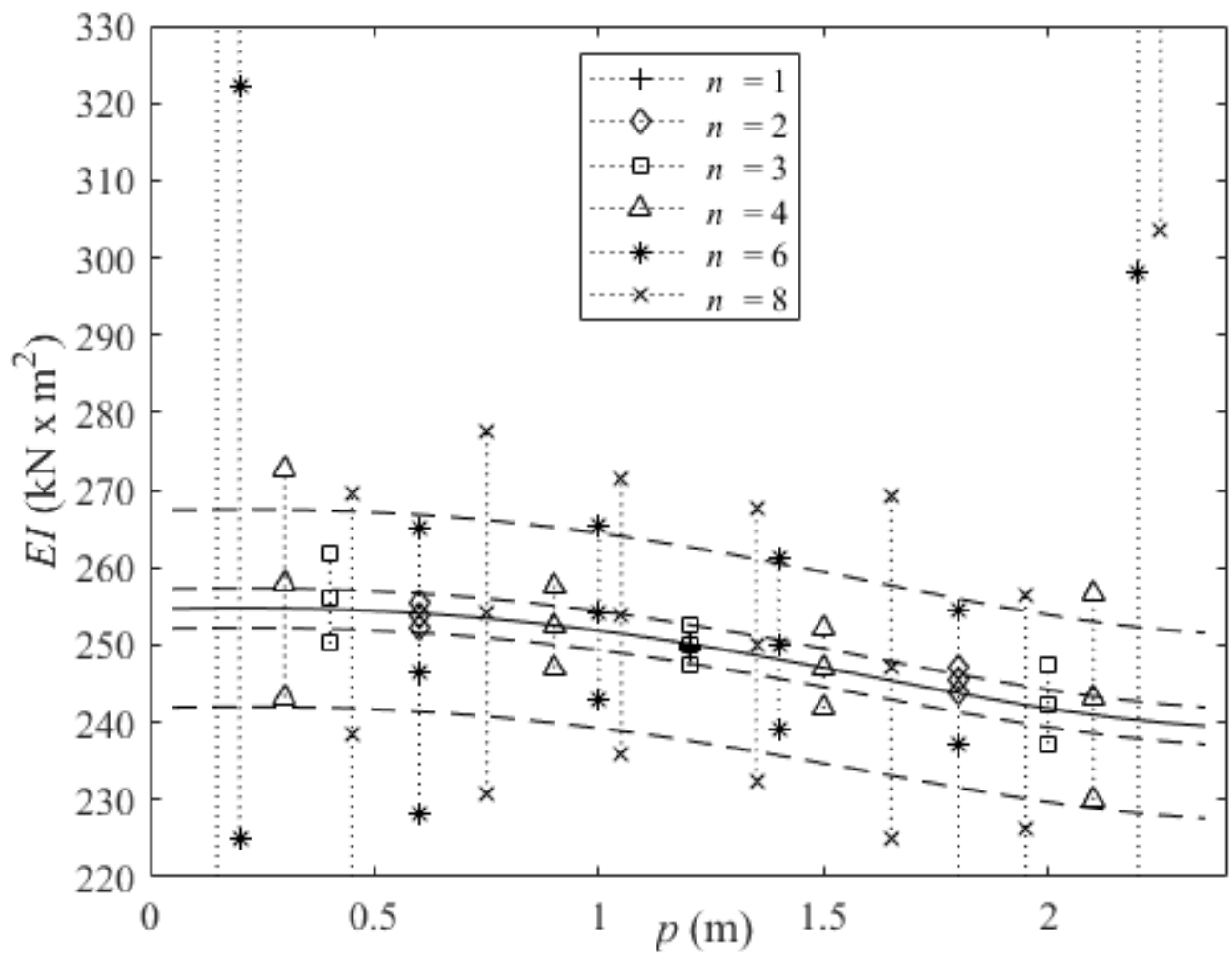

Fig. 13. Stiffness prediction using GCV, deflections and $0.5 \%$ noise

Results for the $1 \%$ and $2 \%$ noise levels shown in Figures 14 and 15 respectively indicate a considerable increase in the error compared to Figures 11 and 12 regardless of the number of unknowns. It can be concluded that rotations show a behaviour that leads to more accurate and reliable stiffness solutions than deflections for the tested scenarios. 


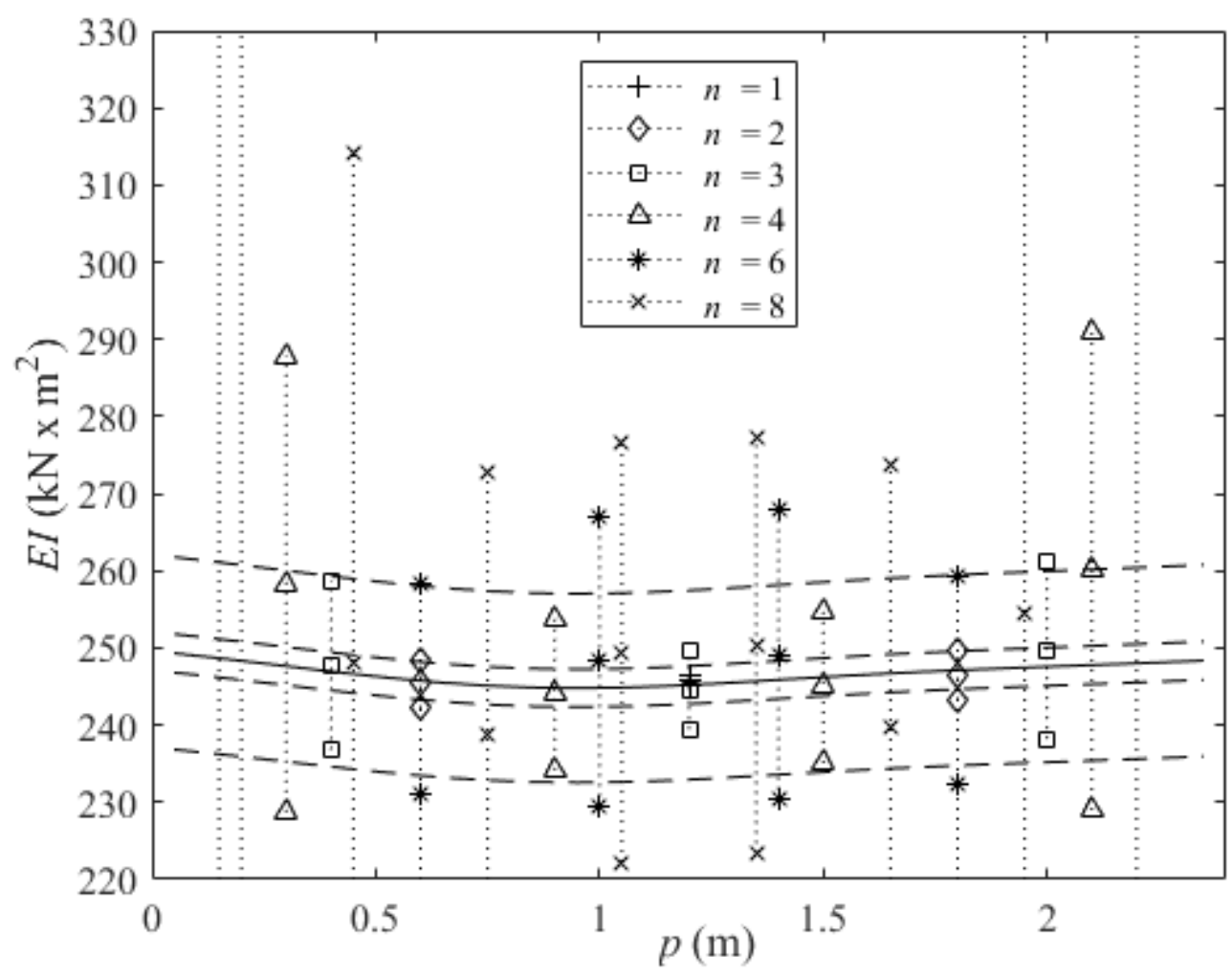

Fig. 14. Stiffness prediction using GCV, deflections and $1 \%$ noise

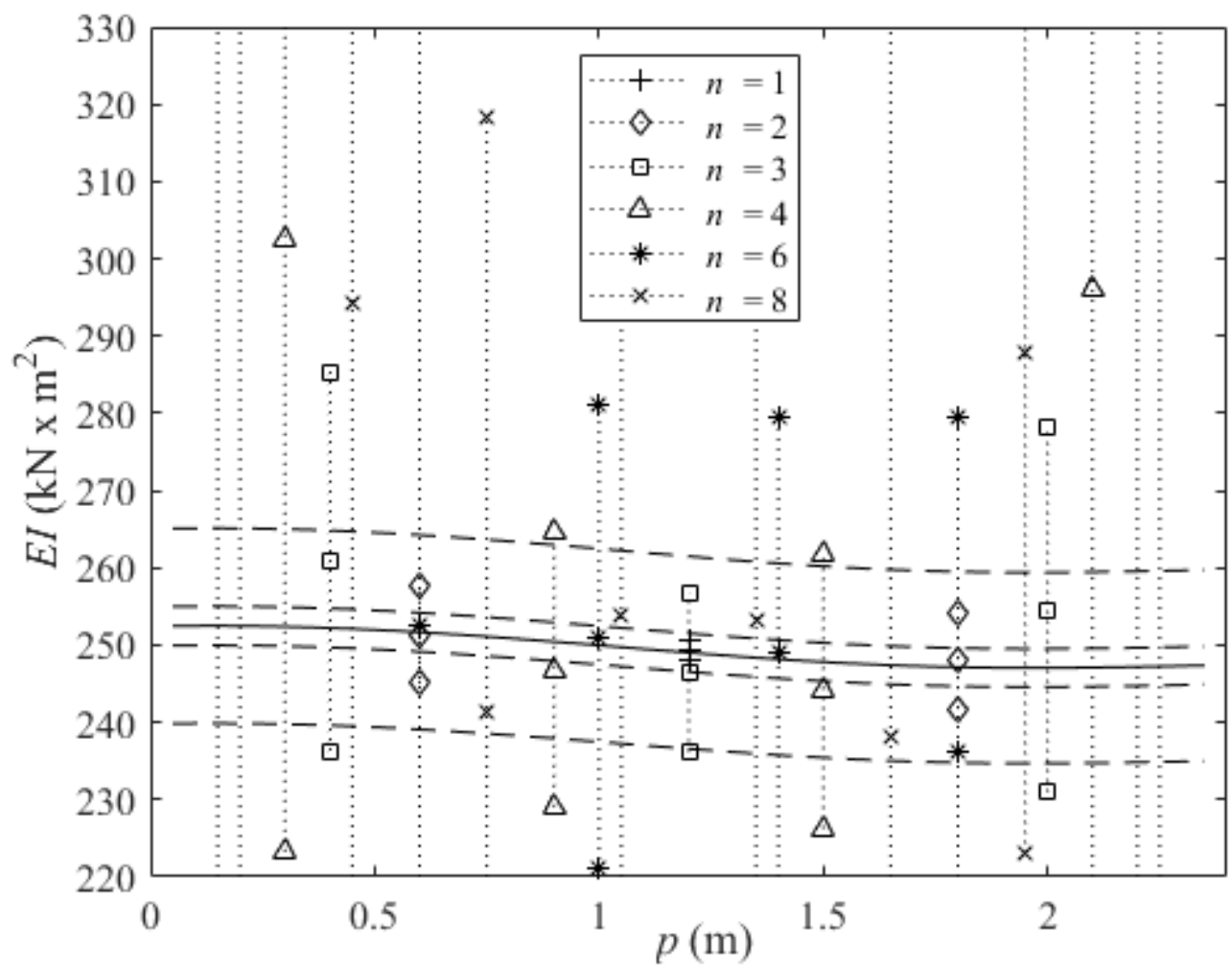

Fig. 15. Stiffness prediction using GCV, deflections and $2 \%$ noise 
The analysis of the results consistently shows that prediction of stiffness for the inner elements is more accurate than that of elements closer to the support, both in the case of deflections and rotations. The reason behind the inaccurate prediction of stiffness close to the supports is explained by the different contribution of each element to deflection at different measurement locations. This contribution is based on the terms of Equation (1) and illustrated by Figure 16 for the case of deflections and $n=12$.

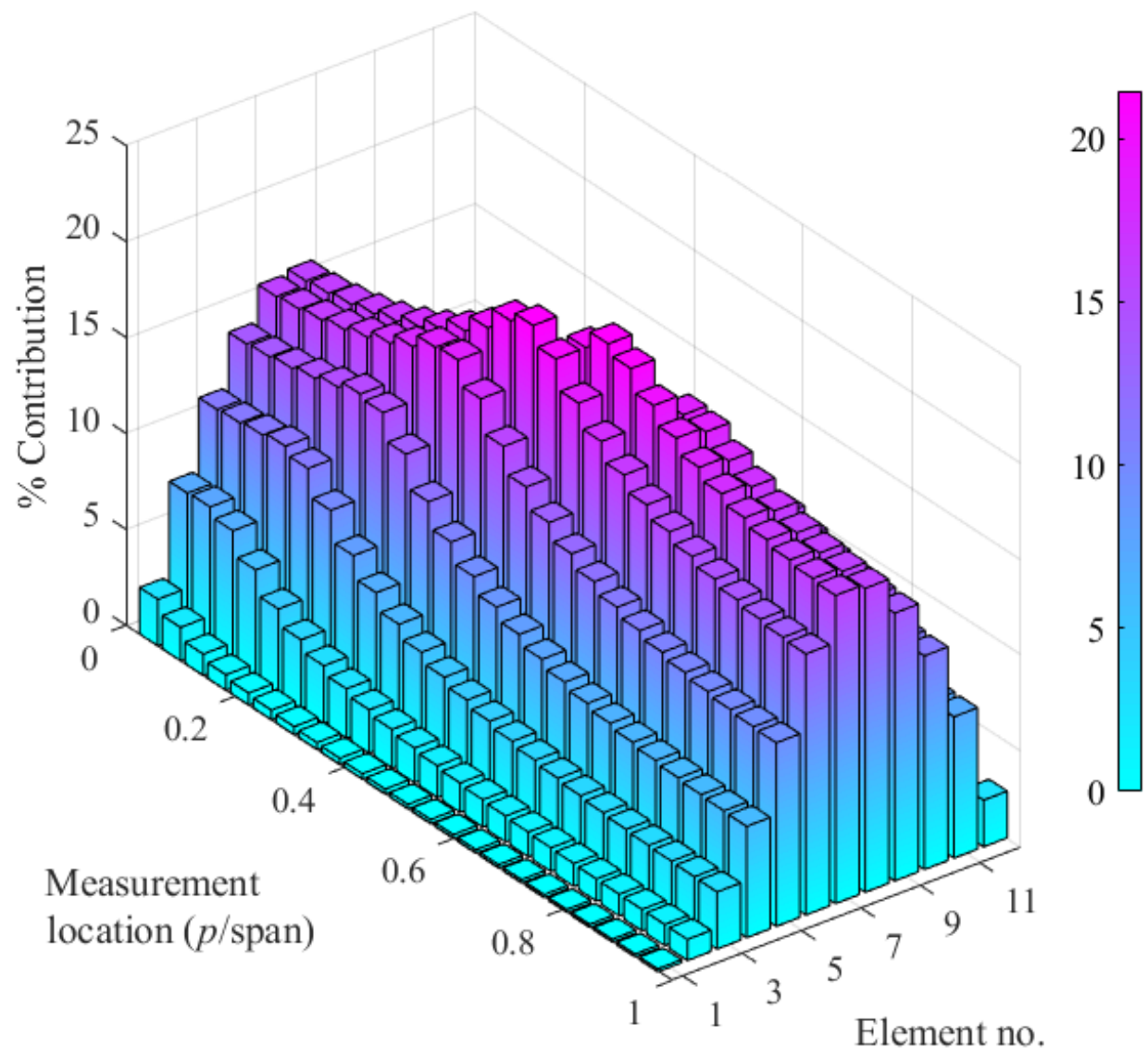

Fig. 16. Contribution of each element to deflections at different locations

The figure shows how the contribution to deflection of the element no. 1, i.e., the one closest to the support, is greater the smaller the distance between the measurement location and the element (i.e., $p /$ span $=0$ ), but barely greater than zero. In contrast, the contribution of the inner elements has a significant weight on the deflections measured all along the discretized beam. 


\section{Results for a damaged beam}

In order to test the methodology in a case with a non-smooth profile, damage is introduced in the stiffness profile previously analysed for the case when rotations with $1 \%$ noise are used as input data. Damage in the beam is assumed to be a crack that causes a variation of stiffness around its location as proposed by Sinha et al (2002). The crack is assumed to have a depth equal to $20 \%$ of the beam height, and to be located at $0.9 \mathrm{~m}$ from the left support. Following Sinha et al, there will be an approximately linear loss of stiffness starting from the crack location and extending $0.165 \mathrm{~m}$ to each side. For the current finite element beam model used in the simulations, the stiffness of four elements is affected with respect to the smooth profile. The two elements closest to the crack suffer a $34 \%$ loss of stiffness, and the remaining two adjacent elements have a $10 \%$ stiffness loss. Figure 17 shows the predicted stiffness when 6 and 8 unknowns are considered; lower values of the number of unknowns have been omitted for clarity since they are unable to capture narrow damages. This figure can be compared to Figure 11 , also based on rotations with $1 \%$ noise, but corresponding to a relatively smooth profile and 5000 simulations, as opposed to the sudden stiffness change and 100 simulations used to obtain Figure 17. Nonetheless, the small standard deviation values of the predictions for inner elements imply that a larger number of simulations would not significantly modify the results. A drop of stiffness is clearly found at around the location of the simulated damage. However, there is a loss of accuracy in the prediction of stiffness in comparison to the case of a healthy beam (Figure 11). Some predictions for the mean stiffness of inner elements, in addition to those closest to the supports, fall now outside the $5 \%$ error range marked by the outer dashed lines in the figure. 


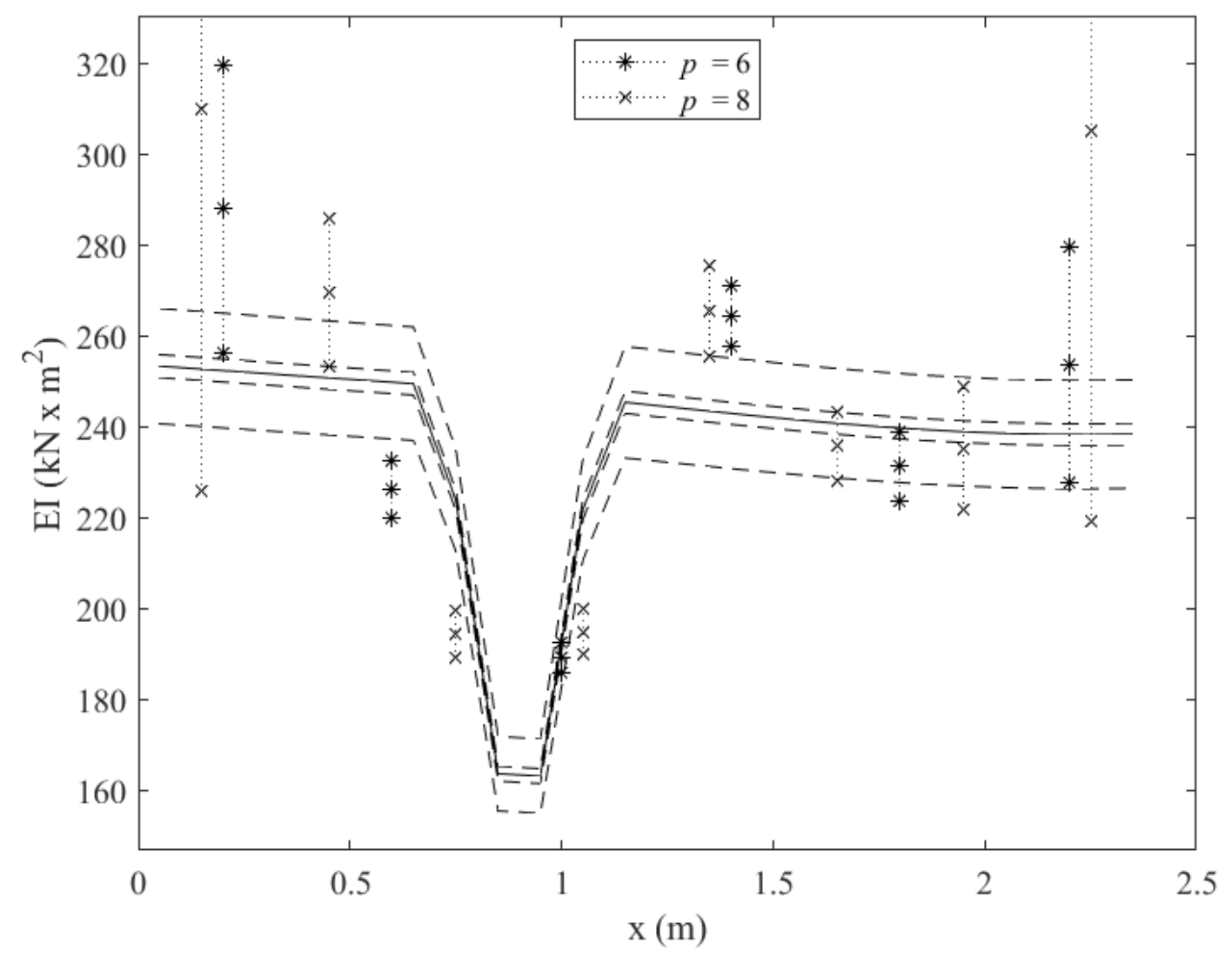

Fig. 17. Stiffness prediction using GCV, rotations and $1 \%$ noise for a damaged beam

\section{Testing using rotations with different levels of noise and number of unknowns in a}

\section{statically indeterminate beam}

In the case of a statically indeterminate beam, the stiffness-displacement problem cannot be linearized. Hence, it is not possible to directly apply the methodology described in the previous sections, and it is necessary to include an optimization algorithm in the process. In particular, the cross-entropy algorithm (Walsh and González 2009, Walsh et al 2010) is used here. The cross-entropy algorithm is a statistical optimization algorithm in which the unknown of the problem is treated as a random variable. Values of that unknown are randomly sampled from a normal distribution to generate several trial (theoretical) beams. Then, the top trial beams 
leading to responses closest to the measures are selected to update the distributions for the next iteration. The process is repeated until convergence is achieved, i.e., the standard deviation of the distributions cannot be further decreased in successive iterations. In the problem at hand, the bending moment at the supports of the fixed-fixed beam is the unknown that is being optimized by the cross-entropy algorithm. Based on the loading and smooth profiles presented in the previous sections, this section assumes that the bending moments at both supports of the fixed-fixed beam should adopt similar values. Although a small inaccuracy is introduced with this simplification, only one random variable needs to be optimized by the cross-entropy algorithm. Clearly, the bending moment at both supports will be different in a general case with a non-smooth profile and non-symmetrical loading. Then, two distinct random variables will have to be considered by the cross-entropy algorithm.

The fixed-fixed beam is solved as the superposition of two loading states of a simply supported beam: (i) the bending moment of a simply supported beam subjected to the same external forces applied to the fixed-fixed beam, and (ii) the bending moment of a simply supported beam subjected to external moments acting at the end supports. The goal of the algorithm is to find a combination of stiffness profiles and external moments at the supports that will provide displacements similar to the target displacements. The optimal solution should take place when the external moments at the supports of the simply supported beam match the moment reactions in the fixed-fixed beam.

The main steps of the cross-entropy algorithm combined with regularization are:

1) Several values $\left(N_{T B}\right)$ of the bending moment at the supports $\left(M_{b}{ }^{j}\right)$ are randomly sampled from a normal distribution $\mathrm{N}\left(\mu_{M b, 0}, \sigma_{M b, 0}\right)$ with initial mean $\mu_{M b, 0}$ and initial standard deviation $\sigma_{M b, 0}$. This sampling allows defining $N_{T B}$ different loading cases (ii) $\left(M_{b}{ }^{1}, M_{b}{ }^{2}\right.$, $\left.\ldots, M_{b}{ }^{j}, \ldots, M_{b}{ }^{N_{T B}}\right)$ for $N_{T B}$ trial beams of unknown stiffness profile. 
2) For each of the $N_{T B}$ trial beams, the methodology to select a regularization parameter described in previous sections is applied to obtain the stiffness profile corresponding to the simply supported beam subjected to both the real loading (loading case (i)) and the bending moments $M_{b}{ }^{j}$ (loading case (ii)).

3) The top trial beams resulting in the displacements closest to the target measures are retained. Each of these top beams will be associated with a loading case (ii) defined by $M_{b}{ }^{j}$

4) The mean and standard deviation of the $M_{b}{ }^{j}$ of the top trial beams are computed and used to update the normal distribution $\mathrm{N}\left(\mu_{M b, 1}, \sigma_{M b, 1}\right)$. This distribution is then used to generate new $N_{T B}$ loading cases (ii) on new $N_{T B}$ trial beams.

5) Steps 2) to 4) are repeated in an iterative process until convergence is achieved. Convergence is said to occur when at an iteration $k$, the rate of change over the previous 10 iterations of the mean $\mu_{M b, k}$ falls below a threshold value.

Simulations have been conducted for a fixed-fixed beam under the same loading conditions as the simply supported beam. For the purpose of comparison, the stiffness profile used in the simply supported beam has been scaled for the fixed-fixed beam, so the deflection at midspan is the same for both boundary conditions. The latter implies that stiffness values used in the fixed-fixed beam are $25 \%$ of those previously analysed for simply supported beams. The number of trial beams $\left(N_{T B}\right)$ for the cross-entropy algorithm is set at 100 , while $10 \%$ is retained as top trial beams to update the normal distributions. In addition, the convergence threshold is assumed to be $1 \%$. GCV is the regularization tool and rotations are used as input data.

Figure 18 shows an average of the results obtained from 5 simulations with $0.5 \%$ noise. The stiffness prediction is less accurate for the fixed-fixed beam than for the simply supported beam shown in Figure 10, but errors are still relatively low. For instance, mean errors remain within the $5 \%$ range except for $0.3 \mathrm{~m}$ to $0.9 \mathrm{~m}$ when $n=8$. It is possible that the results could be 
further improved by increasing the number of simulations and by refining the algorithm to account for different bending moments at both supports of the beam. Moreover, there is a degree of uncertainty associated with the cross-entropy algorithm that could be reduced by conducting several runs of the algorithm for the same simulation.

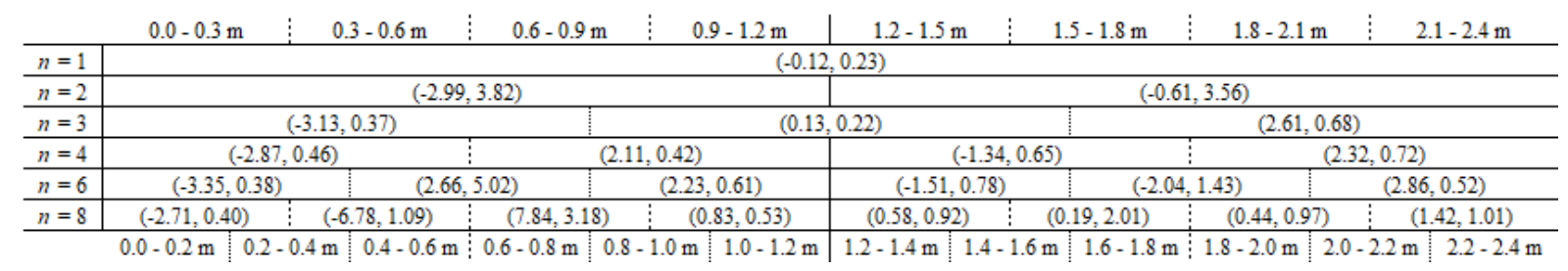

Fig. 18. Statistical parameters $(\mu, \sigma)$ of percentage error in stiffness of a fixed-fixed beam using rotations and $0.5 \%$ noise

Figures 19 (1\% noise) and 20 (2\% noise) for the fixed-fixed beam can be compared to Figures 11 (1\% noise) and 12 (2\% noise) for the simply supported beam. Similarly to Figure 18 , values in Figures 19 and 20 correspond to the average of 5 simulations. While the largest errors in the simply supported beam are associated with elements close to the supports, the largest errors in the fixed-fixed beam occur for elements around a quarter of the length of the beam. The latter is somehow related to the new boundary conditions: Moments are zero at the supports for a simply supported beam, and around the quarter length for the fixed-fixed beam under investigation, i.e., variations in the stiffness of elements in these regions will have a small impact on the measured response. 


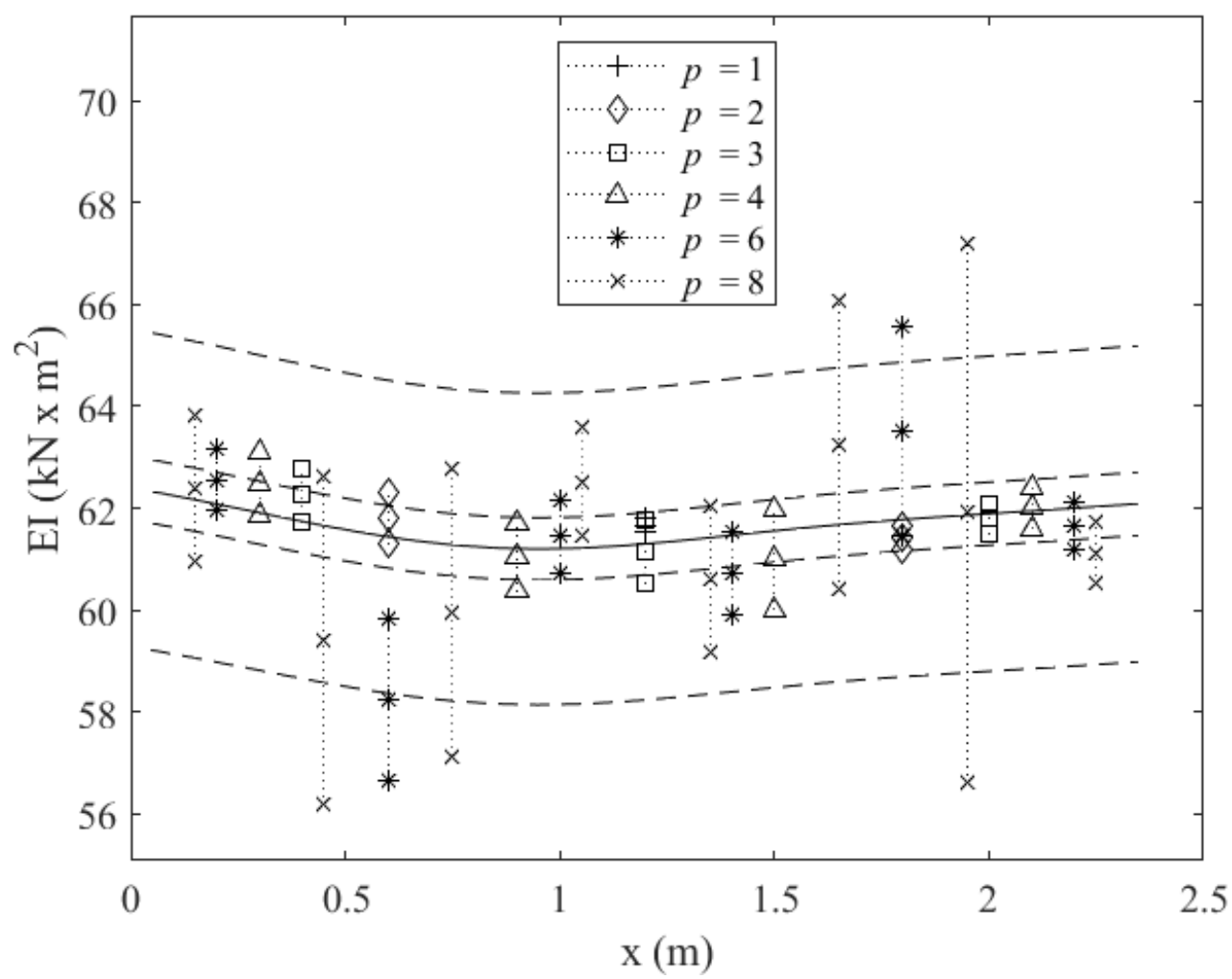

Fig. 19. Stiffness prediction using GCV, rotations and $1 \%$ noise for a fixed-fixed beam

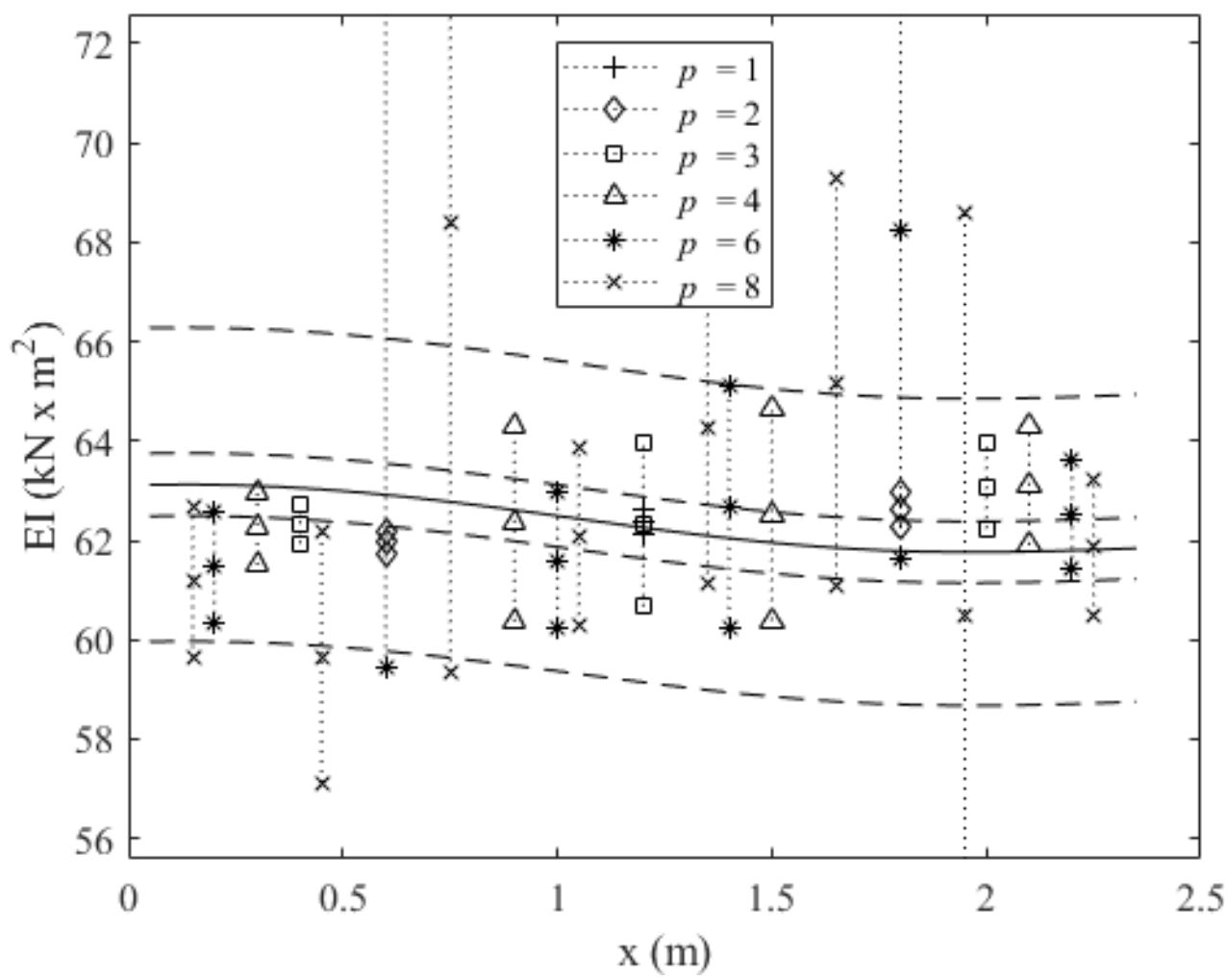

Fig. 20. Stiffness prediction using GCV, rotations and $2 \%$ noise for a fixed-fixed beam 


\section{Conclusions}

This paper has applied regularization tools to improve the prediction of stiffness from the static response of a beam (deflections and rotations) in the presence of noise Regularization methods are often limited by the uncertainty surrounding the selection of the optimal regularization parameter and the associated accuracy of the results. This paper has established a procedure to determine whether a regularization method is definitively unreliable and unable to cope with inaccuracies resulting from the model employed or the noise in the data.

For testing purposes, the theoretical response of a simply supported beam due to a 4-point flexural experiment has been simulated and corrupted with noise. The stiffness profile has been generated based on a relatively smooth spatial correlation function. Recommendations have been provided to choose the regularization parameter that will avoid thresholds including unrealistic negative values. The proposed methodology based on the L-curve or the GCV error function has been shown to predict stiffness along the beam with a reasonable degree of accuracy, except for locations near the supports. Similar results have been obtained when introducing a sudden stiffness drop into the profile. Rotations and GCV have outperformed deflections and L-curve, respectively, for minimizing the average error in the prediction of stiffness. Furthermore, errors have become more significant when the number of unknown stiffness has been increased with respect to the number of measures.

The need to keep the number of unknowns below a reduced number of discrete measures can be a limitation of the methodology in the case of searching for a small drop in stiffness. However, the latter will soon be overcome with the fast development and more affordable costs of modern digital cameras and laser scanner technology able to accurately capture readings along a continuous line or surface. Finally, the profile of a fixed-fixed beam subjected to the 
same previous loading has been successfully assessed by the methodology, although further research is needed to address how rougher profiles and non-symmetrical loading may affect the accuracy of the results. 


\section{Data Availability Statement}

Some or all data, models, or code generated or used during the study are available from the corresponding author by request (MATLAB codes and data).

\section{Acknowledgements}

The authors would like to express their gratitude to the Spanish Government for their financial support towards this research under the Grant No. BIA2011-26915.

\section{References}

Abdo, M.A.-B. (2012). "Parametric study of using only static response in structural damage detection.” Eng. Struct., 34, 124-131.

Bakhtiari-Nejad, F., Rahai, A., and Esfandiari, A. (2005). "A structural damage detection method using static noisy data." Eng. Struct., 27(12), 1784-1793.

Boumechra, N. (2017). "Damage detection in beam and truss structures by the inverse analysis of the static response due to moving loads." Struct. Control Health. Monit., 24(10), 1-10.

Caddemi, S., and Morassi, A. (2007). "Crack detection in elastic beams by static measurements.” Int. J. Solids Struct., 44, 5301-5315.

Caddemi, S., and Morassi, A. (2011). "Detecting multiple open cracks in elastic beams by static tests." J. Eng. Mech., 137(2), 113-124.

Dewangan, U.K., and Barai, S.V. (2012). "System identification with noisy data." Int. J. Adv. Tech. Civ. Eng., 1(2), 82-87.

Ghali, A., and Neville, A. (2017). Structural Analysis: A Unified Classical and Matrix Approach, CRC Press, London, U.K. 
Golub, G.H., Heath, M., and Wahba, G. (1979). "Generalized cross-validation as a method for choosing a good ridge parameter.” Technometrics, 21(2), 215-223.

González, A., Covián, A., Casero, M., and Cooper, J. (2013). "Experimental testing of a crossentropy algorithm to detect damage.” Key Eng. Mater., 569-570, 1170-1178.

González-Jorge, H., Solla, M., Armesto, J., and Arias, P. (2012). "Novel method to determine laser scanner accuracy for applications in civil engineering." Optica Aplicata, 1, 43-53.

Greco, A., Pluchino, A., Caddemi, S., Caliò, I., and Cannizzaro, F. (2019) “On profile reconstruction of Euler-Bernoulli beams by means of an energy based genetic algorithm." Eng. Comp., http://dx.doi.org/10.1007/s00366-018-00693-x.

Ha, D.W., Park, H.S., Choi, S.W., and Kim, Y. (2013). “A wireless MEMS-based inclinometer sensor node for structural health monitoring." Sensors, 13(12), 16090-16104.

Hajializadeh, D., OBrien, E., and Stewart, M.G. (2016). "The sensitivity of bridge safety to spatial correlation of load and resistance." Struct., 5, 23-34.

Hansen, P.C. (1992). “Analysis of discrete ill-posed problems by means of the L-curve.” SIAM Review, 34(4), 561-580.

Hansen, P.C., and O'Leary, D.F. (1993). "The use of the L-curve in the regularization of discrete ill-posed problems.” SIAM J. Sct. Comput., 14(6), 1487-1503.

Hester, D., and González, A. (2015). “A bridge-monitoring tool based on bridge and vehicle accelerations." Struct. Infrastruct. Eng., 11(5), 619-637.

Hjelmstad, K., and Shin, S. (1997). "Damage detection and assessment of structures from static response.” J. Eng. Mech., 123(6), 568-576.

Khuc, T., and Catbas, F.N. (2017). "Completely contactless structural health monitoring of real-life structures using cameras and computer vision." Struct. Control Health Monit., 24, e1852. 
Ma, Q., and Solís, M. (2017). "Damage localization and quantification in simply supported beams using static test data." J. Phys. Conf. Ser., 842(1), 12007.

Ma, Q., and Solís, M. (2019). "Multiple Damage Identification in Beams from Full-Field Digital Photogrammetry.” J. Eng. Mech., 145(8), 04019054.

Mosalam, K.M., Takhirov, S.M., and Park, S. (2014). "Applications of laser scanning to structures in laboratory tests and field surveys." Struct. Control Health Monit., 21, 115-134.

Ok, S.Y., Jung, S., and Song, J. (2018). "Multiobjective optimization approach for robust bridge damage.” Shock Vib., Article ID 3024209.

Pera, D., and I. Ferrando. 2017. “An integrated monitoring system through 3D laser scanner and traditional instruments for load test on arch bridge." Int Arch Photogramm Remote Sen. Spatial Inf Sci. XLII-5/W1: 321-328, https://doi.org/10.5194/isprs-archives-XLII-5-W1-321$\underline{2017}$.

Sanayei, M., and Scampoli, S. (1991). "Structural element stiffness identification from static test data." J. Eng. Mech., 117(5), 1021-1036.

Sinha, J.K., Friswell, M.I., and Edwards, S. (2002). "Simplified models for the location of cracks in beam structures using measured vibration data." J. Sound Vib., 251(1), 13-38.

Sztubecki, J., Bujarkiewicz, A., and Sztubecka, M. (2016). "Measuring displacements in engineering structures by means of a coordinate laser station." Civil Env. Eng. Reports, 23(4), 145-160.

Terlaje, A.S., and Truman, K.Z. (2007). "Parameter identification and damage detection using structural optimization and static response data." Adv. Struct. Eng., 10(6), 607-621.

Tomas, D., Lozano-Galant, J.A., Ramos, G., and Turmo, J. (2018). "Structural system identification of thin web bridges by observability techniques considering shear deformation." Thin-Walled Struct., 123, 282-293. 
Walsh, B., and González, A. (2009). "Assessment of the condition of a beam using a static loading test.” Key Eng. Mater., 413-414, 269-276.

Walsh, B., González, A., and Cantero, D. (2010) “Application of the cross-entropy method to estimate stiffness distribution in plate-type structures." Proc., 10th Int. Conf. on Computational Structures Technology, CST2010, Topping, J.M. Adams, F.J. Pallares, R. Bru, M.L. Romero, eds., Civil-Comp Press, Stirlingshire, UK.

Yang, Q., and Sun, B. (2010). "Structural damage localization and quantification using static test data." Struct. Health Monit., 10(4), 381-389. 